\title{
The Determination of 10 Compounds of GuiZhi Decoction in Rat Plasma after Oral Administration by HPLC-MS/MS and its Application to a Pharmacokinetic Study
}

\section{Huan Gao}

China Academy of Chinese Medical Sciences

Qin Guo

China Academy of Chinese Medical Sciences

Lishi Zhang

China Academy of Chinese Medical Sciences

Jiannan Song

China Academy of Chinese Medical Sciences

Dong Bai ( $\sim$ baidong2000@126.com )

China Academy of Chinese Medical Sciences, Beijing https://orcid.org/0000-0001-6129-9550

\section{Xiaoxia Wu}

China Academy of Chinese Medical Sciences

\section{Research}

Keywords: Guizhi Decoction, HPLC-MS/MS, Multiple Compounds, Pharmacokinetics, Rat Plasma

Posted Date: March 2nd, 2021

DOI: https://doi.org/10.21203/rs.3.rs-254154/v1

License: (c) (i) This work is licensed under a Creative Commons Attribution 4.0 International License.

Read Full License 


\title{
The determination of 10 compounds of GuiZhi Decoction in rat plasma after oral administration by HPLC-MS/MS and its application to a pharmacokinetic study
}

\author{
Huan Gao ${ }^{1,2,+}$, Qin Guo ${ }^{1,+}$, Lishi Zhang ${ }^{1}$, Jiannan Song ${ }^{1}$, Dong Bai ${ }^{1}$ ", and Xiaoxia Wu ${ }^{3}$ *
}

\begin{abstract}
Background: Guizhi Decoction (GZD), a traditional Chinese medical formula, has been commonly used to treat fever, sweating, and cold in China.

Methods: The high performance liquid chromatography-tandem mass spectrometry (HPLCMS/MS) method was established for the determination of 10 compounds, including cinnamic acid, paeoniflorin, albiflorin, liquiritin, isoliquiritin, liquiritigenin, isoliquiritigenin, glycyrrhizic acid, glycyrrhetinic acid, and 6-gingerol. And the specificity, linearity, lower limit of quantification (LLOQ), lower limit of detection (LLOD), precision and accuracy, recovery, matrix effect, and stability were used to verify the HPLC-MS/MS method. This validated method was successfully applied for pharmacokinetic study of the 10 compounds in rat plasma after oral administration of GZD in three doses ( $40 \mathrm{~g}$ crude drug $\cdot \mathrm{kg}^{-1}, 20 \mathrm{~g}$ crude drug $\cdot \mathrm{kg}^{-1}, 10 \mathrm{~g}$ crude drug $\cdot \mathrm{kg}^{-1}$ ) and intravenous injection of GZD extraction at a dose of $2.0 \mathrm{~g}$ crude drug $\mathrm{kg}^{-1}$. The measurements of pharmacokinetic parameters including $\mathrm{AUC}_{0-\infty}, \mathrm{T}_{1 / 2}, \mathrm{~T}_{\max }, \mathrm{C}_{\max }, \mathrm{Vz} \_\mathrm{F}, \mathrm{Cl} \_\mathrm{F}$, and MRT, were performed using a non-compartmental model with Winnonlin 8.1 software.

Results: The results showed that 10 compounds were detected in plasma after oral administration of GZD. the compounds (except for glycyrrhetinic acid) reached the maximum blood concentration quickly, whose Tmax was about 0.1-0.2 min. And a total of 9 compounds were detected after intravenous injection of GZD. The plasma concentration-time curve of these compounds declines rapidly at the beginning, and then decreased slowly, indicating that the plasma concentration-time curves were double exponential function curves.

Conclusions: In this study, the developed method was suitable for pharmacokinetic analysis of the main compounds of GZD in rat plasma, and may reveal the pharmacodynamic material basis of GZD and provide a reference for the rational use of GZD in the clinic.
\end{abstract}

Keywords: Guizhi Decoction; HPLC-MS/MS; Multiple Compounds; Pharmacokinetics; Rat Plasma

\section{Background}

Guizhi Decoction (GZD) is a classical formula from Treatise on Febrile Diseases, a famous ancient Chinese book of traditional Chinese medicine (TCM). Studies have demonstrated that it is mainly used for the treatment of fever, self-sweating, headache, painful stiff nape, and external wind-cold syndrome [1,2]. GZD is composed of five herbal slices, including $9.0 \mathrm{~g}$ of Cinnamomi Ramulus, $9.0 \mathrm{~g}$ of Paeoniae Radix Alba, $9.0 \mathrm{~g}$ of Rhizoma Zingiberis Recens, $9.0 \mathrm{~g}$ of Fructus Jujubae, and $6.0 \mathrm{~g}$ of Radix Glycyrrhizae [3-5]. Modern studies have proven that GZD has bidirectional regulating action on the body temperature, blood pressure, secretion of sweat glands, intestinal peristalsis, and immune function $[6,7]$.

Before this study, we reviewed a large number of pharmacokinetics (PK) studies of the formulae related to GZD. Some researchers have preliminarily discussed the PK characteristics of GZD [4]. There were some PK studies on other Chinese medicine formulae, such as Gualou-Guizhi Decoction,

\footnotetext{
${ }^{*}$ Correspondence: baidong2000@126.com(D. B.); xiaoxia2311@sina.com(X. W.);

${ }^{1}$ Institute of Basic Theory of Traditional Chinese Medicine, China Academy of Chinese Medical Sciences, Beijing, 100700, China

Full list of author information is available at the end of the article.
} 
Guizhi-Gancao Decoction, Guizhi-Fuling Capsule, Huangqi-Guizhi- Wuwu Decoction and so on. For example, 11 compounds in plasma were detected after the rats were given Gualou-Guizhi Decoction by gavage, including liquiritin, isoliquiritin, liquirtin apioside, isoliquiritin apioside, liquiritigenin, soliquiritigenin, glycyrrhizic acid, glycyrrhetinic acid, paeonilorin, albiflorin, and paeonilorin sulfonate. These compounds belonged to Radix Glycyrrhizae and Paeoniae Radix Alba [8]. In the PK study of Guizhi-Gancao Decoction in vivo, 7 compounds were detected, including cinnamaldehyde, cinnamic acid, 2-methoxy cinnamic acid, glycyrrhizic acid, glycyrrhetinic acid, liquiritigenin, and isoliquiritin, from Cinnamomi Ramulus and Radix Glycyrrhizae [9]. 6 compounds in plasma were quantified in the PK study of Guizhi-Fuling Capsul, involving Cinnamomi Ramulus and Paeoniae Radix Alba [10]. Moreover, only two compounds (paeoniorin and astragaloside IV) were analyzed in the PK study of Huangqi-Guizhi-Wuwu decoction [11]. It could be seen that these selected compounds were only from individual herbs in the above studies. These compounds were too few to represent PK activities the whole formula. In addition, after oral administration of TCM formula, some compounds could be transformed into metabolites by enzymes in intestinal flora and liver. These metabolites could be absorbed and have pharmacodynamic effects in vivo. In the previous study, researchers had found that the compounds of GZD absorbed into blood were significantly different from the prototype compounds in decoction by HPLC [12]. We thought only prototype compounds could not represent the real effective ingredients in TCM. There is little doubt that attention should also be paid to metabolites in PK research. In this study, we detected 10 compounds of GZD in rat plasma after intragastric and intravenous administration, including 9 prototype compounds and 1 metabolite.

GZD was composed by five Chinese herbs. In this study, corresponding compounds and their metabolites in each herb were chosen as much as possible, to reveal the overall PK characteristics of GZD. Cinnamic acid was the main compound of Cinnamomi Ramulus, It could combat viral infections, protect neural functions, prevent or slow cognitive decline, and support the immune and digestive systems [13,14]. So, cinnamic acid was selected as the index compounds in Cinnamomi Ramulus. Paeoniflorin and albiflorin were the main glycosides in Paeoniae Radix Alba, and they were isomers of each other. It had been noted that they could transfer to each other by microorganism [15]. Pharmacological studies have exhibited that they had remarkable effects in the treatment of pain, inflammation, muscle spasms, and neurodegenerative disorder properties [16-19]. So, paeoniflorin and albiflorin were selected. The content of gingerol was high in Rhizoma Zingiberis Recens. It has been found to gingerol had a variety of pharmacological effects, such as analgesic, antipyretic, antiemetic, and anti-inflammatory. So, 6-gingerol was selected. Both of liquiritin and isoliquiritin, liquiritigenin and isoliquiritigenin are isomers. These flavonoids compounds had strong therapeutic effects, including anti-inflammation, vasodilation, inhibition of platelet aggregation and regulation of blood lipids. Liquiritin and isoliquiritin were converted into liquiritigenin and isoliquiritigenin in the intestinal tract, which were subsequently metabolized to the glucuronic acid compounds in vivo [20-22]. Glycyrrhetinic acid was the glycoside and metabolite of glycyrrhizic acid. Both of them exhibited high activities, such as antibacterial and anti-inflammatory effects [23-25].So 6 compounds, including 5 prototypes and 1 metabolite, were chosen to be quantitated in Radix Glycyrrhizae.

In this study, a selective HPLC-MS/MS method for the simultaneous determination of multiple compounds of GZD in rat plasma was developed. With this method, the PK process after intragastric and intravenous administration were studied. In this study, we expected that the absorption, metabolism and excretion of GZD in vivo could be systematically studied to reveal the pharmacodynamic process of GZD.

\section{Materials and methods}

\section{Materials and reagents}

Cinnamomi Ramulus, Paeoniae Radix Alba, Rhizoma Zingiberis Recens, Fructus Jujubae and Radix Glycyrrhizae were purchased from Beijing Tongrentang pharmacy (Beijing, China), and were authenticated by Chunsheng Liu, the Professor of Beijing University of Chinese Medicine (Beijing, China), in line with the Pharmacopoeia of the People's Republic of China (Edition 2015) standards.

Cinnamic acid (110,786-201,604), paeoniflorin (110,736-201,741), liquiritin (111,610201,106), glycyrrhetinic acid (110,723-201,514), icariin (110,737-200,415), and aesculetin 
(110,741-200,506) were purchased from National Institutes for Food and Drug Control (Beijing, China). Isoliquiritin (TT102I), liquiritigenin (TT011L), and glycyrrhizic acid (AB291G) were purchased from Epuresino technology (Tianjin, China) Co., Ltd (Tianjin, China). Albiflorin (Y15D8H50784) was purchased from Shanghai yuanye Bio-Technology Co., Ltd (Shanghai, China). 6-Gingerol (ASB-00007164-005) and isoliquiritigenin (ASB-00009265-005) were purchased from ChromaDex, Inc. (Irvine, CA, USA). The purity of all standards was greater than $98 \%$. Acetonitrile was purchased from Fisher Scientific (Hampton, NH, USA). The chemical structures of the analytes and internal standards (ISs) were shown in Fig. 1. Methanol and formic acid were both purchased from Thermo Fisher Scientific-CN (Beijing, China); acetic acid and ammonium acetate were 
purchased from Dikma Technologies Inc. (Beijing, China). All reagents were chromatographically pure.

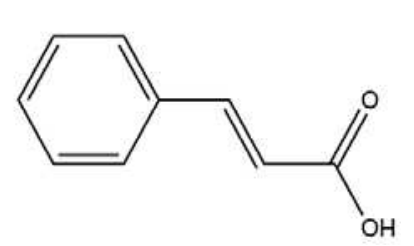

Cinnamic acid

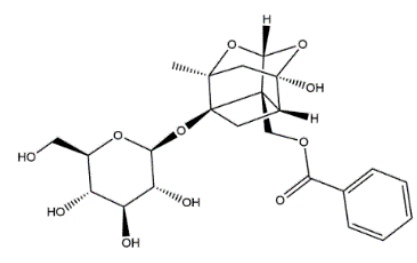

Paeoniforin

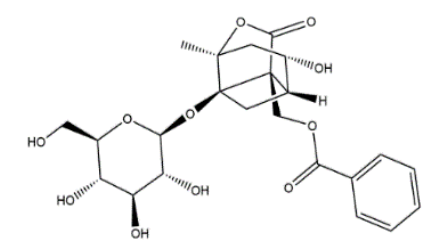

Albiflorin<smiles>O=C1CC(c2ccc(O[C@H]3OC(CO)[C@@H](O)[C@H](O)[C@H]3O)cc2)Oc2cc(O)ccc21</smiles>

Liquiritin

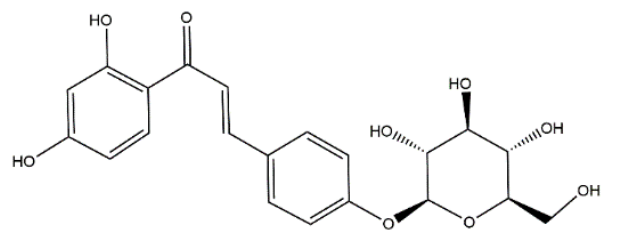

Isoliquiritin

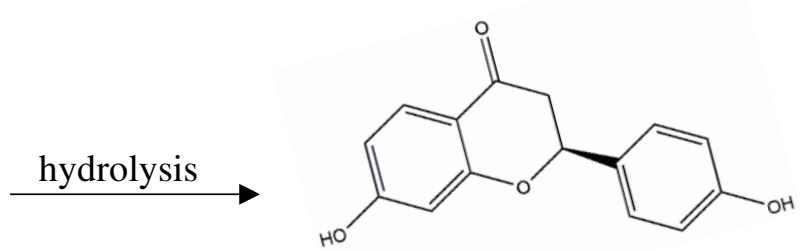

Liquiritigenin

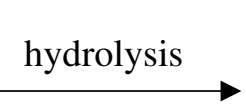

Isoliquiritigenin

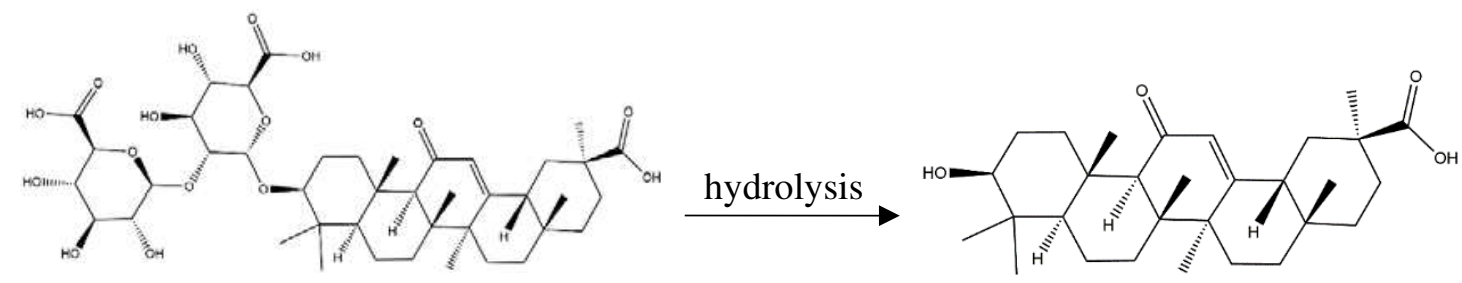

Glycyrrhizic acid<smiles>CCCCCC(O)CC(=O)CCc1ccc(N)c(OC)c1</smiles>

6-Gingerol

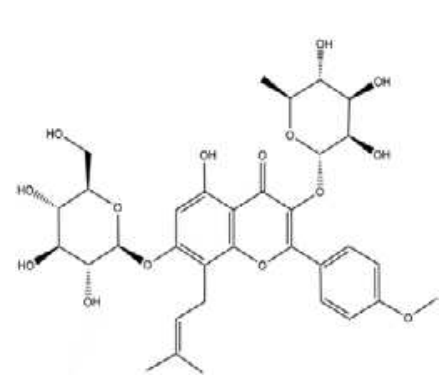

Icariin (IS1)
Glycyrrhetinic acid<smiles>O=c1ccc2cc(O)c(O)cc2o1</smiles>

Aesculetin (IS2)

Fig.1 Chemical structures of analytes and internal standard (IS)

\section{Animals}

30 clean male Sprague-Dawley rats $(300 \pm 20 \mathrm{~g})$ were purchased from SPF (Beijing) Biotechnology Co., Ltd. (Beijing, China quality certification number: SCXK (Jing) 2016-0002). The temperature and humidity of the controlled environmental conditions were $23 \pm 3^{\circ} \mathrm{C}$ and $50 \%$ $\pm 10^{\circ} \mathrm{C}$, respectively. The principles of laboratory animal care and all protocols were in accordance with the relevant national legislation and local guidelines and were approved by Animal Care and 
Use Committee of the Institute of Basic Theory for Chinese Medicine, China Academy of Chinese Medical Sciences (Date: 25 March 2019; No.: SYXK (Jing) 2019-008).

\section{Preparation of GZD for oral adminstration}

The volatile oil of Ramulus Cinnamomiand and Rhizoma Zingiberis Recens was extracted by steam distillation with four folds water for $2 \mathrm{~h}$. The residues of the two herbal slices and other three herbal slices were boiled twice with eight folds water for 30 min each time. All those water decoctions were mixed together and concentrated to $1.0 \mathrm{~g}$ crude drugs per milliliter. After the volatile oil were added and mixed together, the extraction was stored at $4^{\circ} \mathrm{C}$.

\section{Preparation of GZD extraction for injection}

GZD solution $\left(1.0 \mathrm{~g} \cdot \mathrm{mL}^{-1}\right)$ was centrifuged $\left(15,000 \mathrm{rpm}, 15 \mathrm{~min}, 4^{\circ} \mathrm{C}\right)$ and the supernatant was filtered with $0.2 \mu \mathrm{m}$ filter membranes. Then, $100 \mathrm{~mL}$ filtrate was vacuum freeze-dried into powder, which was dissolved with $500 \mathrm{~mL}$ sodium chloride injection $(0.9 \%)$. The solution was filtered with $0.2 \mu \mathrm{m}$ filter membrane in the super clean bench to remove the bacteria and then packed separately and stored at $4^{\circ} \mathrm{C}$. This extraction contained $0.2 \mathrm{~g}$ crude drugs per milliliter.

\section{HPLC-MS/MS conditions}

Agilent 1260 with the Rapid Resolution Liquid Chromatography (RRLC) system (Agilent Technologies, Santa Clara, CA, USA) was used. The separation of compounds was carried out on the Agilent SB C18 column $(2.1 \times 50 \mathrm{~mm}, 1.8 \mu \mathrm{m})$ at $35^{\circ} \mathrm{C}$.

Agilent 6410 mass spectrometry was equipped with Electron Spray Ionization (ESI), using Multiple Reaction Monitoring (MRM) with negative ion mode detection. The ion source temperature was $350^{\circ} \mathrm{C}$; the drying gas was nitrogen $\left(\mathrm{N}_{2}\right.$, purity of $\left.99.9 \%\right)$; the flow rate was 10 $\mathrm{L} \cdot \mathrm{min}^{-1}$; the nebulizing gas pressure was $40 \mathrm{psi}$; and the capillary voltage was $4.0 \mathrm{kv}$. As the analytes and IS had different ionization activities, two optimized mobile phases were used separately to ensure the sensitivity. The MS conditions, including the ion pair, fragmentor voltage, and collision energy were all optimized with standard solutions.

Condition I: the mobile phase was composed of $0.1 \%$ aqueous acetic acid (A) and acetonitrile (B) with a gradient program $(0.0-1.0 \mathrm{~min}, 5 \% \mathrm{~B} ; 1.1-5.0 \mathrm{~min}, 14 \% \mathrm{~B} ; 5.1-8.0 \mathrm{~min}, 20 \% \mathrm{~B} ; 8.1-11.0$ $\min , 45 \% \mathrm{~B}, 11.1-17.0 \mathrm{~min}, 85 \% \mathrm{~B}$ ). Condition II: the mobile phase was composed of $0.2 \%$ acetic acid aqueous solution containing $2 \mathrm{mmoL} \cdot \mathrm{L}^{-1}$ ammonium acetate (A) and acetonitrile (B) with a gradient program $(0.0-1.0 \mathrm{~min}, 5 \% \mathrm{~B} ; 1.1-8.0 \mathrm{~min}, 45 \% \mathrm{~B})$. The flow rate was $0.3 \mathrm{~mL} \cdot \mathrm{min}^{-1}$ and the injection volume was $2 \mu \mathrm{L}$. The stop time was $8.0 \mathrm{~min}$ with the post time of $6.0 \mathrm{~min}$.

\section{Sample preparation}

In this study, the methods between liquid-liquid extraction (LLE) and protein precipitation (PPT) were compared to extract and purify the compounds to be tested in rat plasma. For LLE, ethyl acetate with or without hydrochloric acid were used as the extract liquor. For PPT, methanol and acetonitrile with or without hydrochloric acid were used to precipitate protein. The mixed reference solution was added into the blank plasma samples and prepared with above methods separately and injected into HPLC-MS/MS. The ratio of the peak area of each compound in the plasma sample to that of in the reference solution with same concentration was taken as the extraction rate. The method with a high extraction rate was chosen to prepare the samples.

\section{HPLC-MS/MS method validation}

\section{Specificity}

The specificity of the method was assessed by comparing chromatograms of blank plasma samples from six individual rats, blank plasma spiked with the 10 analytes and 2 ISs. The plasma samples were obtained at 30 min or $4 \mathrm{~h}$ from the rats after oral administration of the GZD or GZD extraction. 
The standard stock solutions of 10 analytes and 2 ISs were prepared in methanol. The initial concentrations of each compounds were $12,750 \mathrm{ng} \cdot \mathrm{mL}^{-1}$ for cinnamic acid, $3248 \mathrm{ng} \cdot \mathrm{mL}^{-1}$ for paeoniflorin, $3488 \mathrm{ng} \cdot \mathrm{mL}^{-1}$ for albiflorin, $3568 \mathrm{ng} \cdot \mathrm{mL}^{-1}$ for 6-gingerol, $3248 \mathrm{ng} \cdot \mathrm{mL}^{-1}$ for liquiritin, $3248 \mathrm{ng} \cdot \mathrm{mL}^{-1}$ for isoliquiritin, $3728 \mathrm{ng} \cdot \mathrm{mL}^{-1}$ for liquiritigenin, $3328 \mathrm{ng} \cdot \mathrm{mL}^{-1}$ for isoliquiritigenin, $3488 \mathrm{ng} \cdot \mathrm{mL}^{-1}$ for glycyrrhizic acid, and $8360 \mathrm{ng} \cdot \mathrm{mL}^{-1}$ for glycyrrhetinic acid. The concentration of 2 ISs were $508 \mathrm{ng} \cdot \mathrm{mL}^{-1}$ for icariin and $638 \mathrm{ng} \cdot \mathrm{mL}^{-1}$ for aesculetin.To prepare the working solutions for calibration samples, 10 series concentrations solutions were prepared by methanol dilution. $20 \mu \mathrm{L}$ working solution was added to the blank plasma.

Plasma calibration curves were constructed using the peak area ratios of the 10 analytes to the ISs, and applying separate weighted $\left(1 / \chi^{2}\right)$ least squares linear regression. The lower limit of quantification (LLOQ) and the lower limit of detection (LLOD) were defined by the signal-to-noise ratio method. LLOQ should be ten times the noise level ( $\mathrm{S} / \mathrm{N} \geq 10$ ), and LLOD should be three times the noise level $(\mathrm{S} / \mathrm{N} \geq 3)$.

\section{Precision and accuracy}

The accuracy and precision of the method were evaluated by intra- and inter-day variations. Standard solutions were added to the blank plasma and prepared at three different concentrations (low, medium, and high). Intra-day precision was evaluated with six replicates at one day, and interday precision was evaluated at three days. The precision of each compound was evaluated by relative standard deviation (RSD) value, which should not exceed $15.0 \%$, and the accuracy was estimated with the relative error (RE), which should be within $\pm 15.0 \%$.

\section{Extraction recovery and matrix Effect}

The recoveries of the analytes from plasma samples were determined by comparing the peak areas of the analytes in plasma samples after extraction to those of the same concentration of the analytes spiked into the solution extracted from plasma samples. The matrix effects were measured by comparing the peak areas obtained from samples with the analytes spiked after extraction, at three concentration levels (low, middle, and high), to those obtained from standard solutions at the same concentrations.

\section{Stability}

Stability of the analytes from the plasma samples were investigated by determining three different concentrations (low, medium and high samples) in five replicates under different storage conditions. The stability in plasma was assessed by analyzing (i) samples kept at room temperature $\left(25^{\circ} \mathrm{C}\right)$ for $24 \mathrm{~h}$, (ii) samples after three freeze-thaw cycles, (iii) samples after stored at $-80^{\circ} \mathrm{C}$ for 15 days.

\section{Pharmacokinetic study}

30 clean male Sprague-Dawley rats $(300 \pm 20 \mathrm{~g})$ were divided into oral administration (ig) groups (high, medium, and low dose), intravenous injection (iv) group and blank group. There were 6 rats in each ig group and 5 rats in iv group. All animals were fasted for $12 \mathrm{~h}$ but with access to water before experiment.

Rats in ig group were given GZD by gavage according to their body weight $\left(4.0 \mathrm{~mL} \cdot 100 \mathrm{~g}^{-1}\right)$. The high dose group was given GZD in a concentration of $1.0 \mathrm{~g}$ crude drug $\cdot \mathrm{mL}^{-1}$, which was about 10 times as much as the clinical dosage ( $40.0 \mathrm{~g}$ crude drug $\left.\cdot \mathrm{kg}^{-1}\right)$. The medium dose group was in a concentration of $0.5 \mathrm{~g}$ crude drug $\cdot \mathrm{mL}^{-1}\left(20.0 \mathrm{~g}\right.$ crude drug $\left.\cdot \mathrm{kg}^{-1}\right)$, and the low dose group was in a concentration of $0.25 \mathrm{~g}$ crude drug $\cdot \mathrm{mL}^{-1}\left(10.0 \mathrm{~g}\right.$ crude drug $\left.\cdot \mathrm{kg}^{-1}\right) .300 \mu \mathrm{L}$ blood samples were collected in Eppendorf tubes from the postorbital venous plexus veins of each rat by capillary tubes before dose $(0 \mathrm{~h})$ and after doses at $5 \mathrm{~min}, 15 \mathrm{~min}, 30 \mathrm{~min}, 1 \mathrm{~h}, 2 \mathrm{~h}, 4 \mathrm{~h}, 8 \mathrm{~h}, 12 \mathrm{~h}$, and $24 \mathrm{~h}$.

Rats in iv group were injected with GZD extraction via the caudal vein according to their body weight $\left(1.0 \mathrm{~mL} \cdot 100 \mathrm{~g}^{-1}\right)$, which was equivalent to 0.5 times as much as the clinical dosage $(2.0 \mathrm{~g}$ crude drug $\cdot \mathrm{kg}^{-1}$ ). Blood samples were collected at $0 \mathrm{~min}, 2 \mathrm{~min}, 15 \mathrm{~min}, 30 \mathrm{~min}, 1 \mathrm{~h}, 2 \mathrm{~h}, 4 \mathrm{~h}, 8 \mathrm{~h}$, $12 \mathrm{~h}$, and $24 \mathrm{~h}$ after injecting GZD extraction.

Rats in the blank group were used to collect blank blood samples from the abdominal aorta after anesthetized by intraperitoneal injection of $3 \%$ sodium pentobarbital in the dosage of $0.5 \mathrm{~mL} \cdot 100$ $\mathrm{g}^{-1}$. 
The blood samples were centrifuged at $12,000 \mathrm{rpm}$ for $10 \mathrm{~min}$ at $4^{\circ} \mathrm{C}$, and stored at $-80^{\circ} \mathrm{C}$ until analysis.

\section{Data analysis}

The validated HPLC-MS/MS methods were applied to analyze the concentrations of 10 compounds in rat plasma after oral or parenteral administration GZD at different times. The pharmacokinetic parameters were obtained by the non-compartmental analysis of plasma concentration versus time data using a non-compartmental model of the Phoenix Winnonlin 8.1 software (Certara, USA).

\section{Results}

\section{Optimization of chromatographic and Mass conditions}

In order to obtain high detection sensitivity and good peak symmetry of the analytes, different ratios of formic acid, acetic acid, or ammonium acetate were chosen as the mobile phase annexing additive. Finally, we found that the separation and detection of the analytes were determined with these two phase conditions. The mobile phase used acetonitrile and $0.1 \%$ formic acid was suitable for paeoniflorin, albiflorin, liquiritin, isoliquiritin, liquiritigenin, isoliquiritigenin, glycyrrhizic acid, glycyrrhetinic acid, and icariin (IS1), while the mobile phase condition of acetonitrile and $0.2 \%$ acetic acid containing $2 \mathrm{mmoL} \cdot \mathrm{L}^{-1}$ ammonium acetate was suitable for cinnamic acid, 6-gingerol, and aesculetin (IS2). The ionization conditions of those compounds, such as capillary voltage, nebulizer pressure, fragmentor voltage, and collision energy, were optimized by using the standard solutions. The optimized ionization conditions for the 10 compounds and 2 ISs were summarized in Table 1.

Table 1 MS/MS parameters of the analytes and internal standards

\begin{tabular}{lllll}
\hline Analytes & $\begin{array}{l}\text { Quantification } \\
\text { Transition } \\
(\boldsymbol{m} / \boldsymbol{z})\end{array}$ & $\begin{array}{l}\text { Dwell Time } \\
(\mathbf{m s})\end{array}$ & $\begin{array}{l}\text { Fragmentor } \\
\text { Voltage } \\
(\mathbf{V})\end{array}$ & $\begin{array}{l}\text { Collision } \\
\text { Energy } \\
(\mathbf{V})\end{array}$ \\
\hline Cinnamic acid & $147.0 \rightarrow 103.0$ & 60 & 62 & 6 \\
Paeoniflorin & $525.2 \rightarrow 121.0$ & 70 & 157 & 37 \\
Albiflorin & $525.2 \rightarrow 121.0$ & 70 & 157 & 37 \\
Liquiritin & $417.2 \rightarrow 255.2$ & 70 & 152 & 15 \\
Isoliquiritin & $417.2 \rightarrow 255.2$ & 70 & 152 & 15 \\
Liquiritigenin & $255.1 \rightarrow 119.0$ & 70 & 105 & 24 \\
Isoliquiritigenin & $255.1 \rightarrow 119.0$ & 70 & 105 & 24 \\
Glycyrrhizic acid & $821.2 \rightarrow 350.9$ & 70 & 181 & 40 \\
Glycyrrhetinic acid & $469.4 \rightarrow 425.5$ & 70 & 190 & 41 \\
6-Gingerol & $293.1 \rightarrow 99.1$ & 60 & 82 & 6 \\
Icariin (IS1) & $721.3 \rightarrow 367.2$ & 60 & 120 & 35 \\
Aesculetin (IS2) & $177.1 \rightarrow 133.0$ & 60 & 110 & 15 \\
\hline
\end{tabular}

\section{Sample pretreatment}

The absolute recoveries of 10 analytes and 2 ISs after different sample pretreatments could be found in Table 2. The final sample treatment method was as follows: $20 \mu \mathrm{L}$ IS solution $\left(500 \mathrm{ng} \cdot \mathrm{mL}^{-1}\right.$ of icariin; $600 \mathrm{ng} \cdot \mathrm{mL}^{-1}$ of aesculetin) and $10 \mu \mathrm{L}$ hydrochloric acid $\left(20 \mathrm{mmol} \cdot \mathrm{L}^{-1}\right)$ were added into each $100 \mu \mathrm{L}$ plasma sample. The sample was mixed for 1 min and $200 \mu \mathrm{L}$ methanol was added. Then the mixture was vortex for $3 \mathrm{~min}$. The sample was centrifuged at 12,000 rpm for $15 \mathrm{~min}$ at $4^{\circ} \mathrm{C} .300 \mu \mathrm{L}$ of the supernatant was collected and evaporated to dryness by vacuum freeze-drying. Finally, the residue was dissolved by $100 \mu \mathrm{L}$ mobile phase (ACN: $\mathrm{H} 2 \mathrm{O}=1: 1)$ and vortexed for 3 min. After centrifuged at $12,000 \mathrm{rpm}$ for $15 \mathrm{~min}$ at $4^{\circ} \mathrm{C}, 2 \mu \mathrm{L}$ aliquot was injected into HPLCMS/MS system. 
Table 2 Absolute recoveries of the analytes and internal standards after different sample pretreatments methods $(n=3$, mean \pm SD).

\begin{tabular}{|c|c|c|c|c|c|c|c|c|c|c|c|c|}
\hline Analytes & $\begin{array}{l}\text { Ethylacetate } \\
(\%)\end{array}$ & $\begin{array}{l}\text { Ethylacetate + } \\
50 \mathrm{mmol} \cdot \mathrm{L}^{-1} \\
\mathrm{HCl}(\%)\end{array}$ & $\begin{array}{l}\mathrm{MeOH} \\
(\%)\end{array}$ & $\begin{array}{l}\mathrm{MeOH}+50 \\
\mathrm{mmol} \cdot \mathrm{L}^{-1} \\
\mathrm{HCl}(\%)\end{array}$ & $\begin{array}{l}\mathrm{MeOH}+20 \\
\mathrm{mmol} \cdot \mathrm{L}^{-1} \\
\mathrm{HCl}(\%)\end{array}$ & $\begin{array}{l}\mathrm{MeOH}+10 \\
\mathrm{mmol} \cdot \mathrm{L}^{-1} \\
\mathrm{HCl}(\%)\end{array}$ & $\begin{array}{l}\mathrm{MeOH}+5 \\
\mathrm{mmol} \cdot \mathrm{L}^{-1} \\
\mathrm{HCl}(\%)\end{array}$ & $\begin{array}{l}\text { ACN } \\
(\%)\end{array}$ & $\begin{array}{l}\mathrm{ACN}+50 \\
\mathrm{mmol} \cdot \mathrm{L}^{-1} \\
\mathrm{HCl}(\%)\end{array}$ & $\begin{array}{l}\mathrm{ACN}+20 \\
\mathrm{mmol} \cdot \mathrm{L}^{-1} \\
\mathrm{HCl}(\%)\end{array}$ & $\begin{array}{l}\mathrm{ACN}+10 \\
\mathrm{mmol} \cdot \mathrm{L}^{-1} \\
\mathrm{HCl}(\%)\end{array}$ & $\begin{array}{l}\mathrm{ACN}+5 \\
\mathrm{mmol} \cdot \mathrm{L}^{-1} \\
\mathrm{HCl}(\%)\end{array}$ \\
\hline Cinnamic acid & $8.0 \pm 0.5$ & $107.4 \pm 8.5$ & $102.2 \pm 8.6$ & $108.0 \pm 11.7$ & $106.8 \pm 2.4$ & $101.0 \pm 2.7$ & $103.6 \pm 8.7$ & $108.3 \pm 11.9$ & $100.3 \pm 12.9$ & $108.7 \pm 17.1$ & $106.9 \pm 15.1$ & $105.3 \pm 12.4$ \\
\hline & & & & & & & & & & & & \\
\hline Albiflorin & $.5 \pm 0.7$ & $2.5 \pm 8.9$ & $62.9 \pm 13.2$ & $106.6 \pm 12.1$ & $111.5 \pm 2.3$ & $117.9 \pm 1.1$ & $100.6 \pm 7.4$ & $73.0 \pm 3.5$ & $96.4 \pm 16.7$ & $120.1 \pm 10.6$ & $9.5 \pm 10.8$ & $118.8 \pm 2.1$ \\
\hline & & & & $92.9 \pm 8.7$ & & & $.5 \pm 14.4$ & $116.4 \pm 17.6$ & & & & $5.2 \pm 15.0$ \\
\hline Isoliquiritin & $3 \pm 6.7$ & $2.8 \pm 8.6$ & $61.3 \pm 10.7$ & $94.5 \pm 10.4$ & $100.2 \pm 1.9$ & $102.1 \pm 1.8$ & $95.8 \pm 6.6$ & $62.7 \pm 16.8$ & $96.6 \pm 10.0$ & $106.8 \pm 19.6$ & $82.7 \pm 12.8$ & $103.5 \pm 10.4$ \\
\hline Liquiritigenin & $3.9 \pm 7.7$ & $3.2 \pm 8.6$ & $76.2 \pm 12.3$ & $97.3 \pm 0.1$ & $107.3 \pm 1.3$ & $110.9 \pm 1.0$ & $102.5 \pm 11.8$ & $75.6 \pm 15.6$ & $98.1 \pm 9.4$ & $112.0 \pm 8.3$ & $108.7 \pm 11.7$ & $114.5 \pm 16.5$ \\
\hline Isoliqu & & & & & & & & & $115.8 \pm 10.5$ & $126.6 \pm 17.6$ & & $113.8 \pm 2.5$ \\
\hline Glycyrrhizic acid & $0.0 \pm 0.0$ & $0.0 \pm 0.0$ & $101.8 \pm 17.2$ & $12.3 \pm 0.6$ & $52.6 \pm 5.0$ & $59.9 \pm 0.9$ & $63.5 \pm 1.3$ & $77.9 \pm 1.9$ & $17.2 \pm 23.6$ & $44.1 \pm 9.6$ & $64.4 \pm 14.6$ & $63.2 \pm 4.1$ \\
\hline Glycyrrhetinic acid & $36.0 \pm 7.1$ & $48.7 \pm 4.4$ & $36.2 \pm 6.9$ & $55.2 \pm 7.7$ & $59.4 \pm 1.2$ & $58.6 \pm 1.5$ & $59.0 \pm 9.6$ & $39.3 \pm 9.4$ & $63.2 \pm 14.3$ & $64.6 \pm 3.6$ & $61.7 \pm 4.3$ & $65.6 \pm 15.1$ \\
\hline 6-Gingerol & $130.0 \pm 17.6$ & $6.0 \pm 4.2$ & $84.2 \pm 12.4$ & $102.3 \pm 8.7$ & $100.4 \pm 14.8$ & $111.7 \pm 1.9$ & $101.4 \pm 7.8$ & $89.2 \pm 7.7$ & $100.3 \pm 11.7$ & $108.5 \pm 12.9$ & $100.0 \pm 13.5$ & $108.7 \pm 12.4$ \\
\hline IS1 & $94.2 \pm 9.6$ & $89.2 \pm 10.8$ & $72.1 \pm 12.1$ & $94.1 \pm 6.0$ & $114.4 \pm 12.8$ & $120.2 \pm 2.6$ & $105.9 \pm 15.0$ & $82.2 \pm 13.1$ & $104.0 \pm 8.5$ & $120.2 \pm 17.5$ & $114.1 \pm 10.0$ & $124.4 \pm 7.6$ \\
\hline IS2 & $80.1 \pm 10.2$ & $2.5 \pm 0.0$ & $51.3 \pm 4.0$ & $98.3 \pm 5.7$ & $82.0 \pm 1.2$ & $62.6 \pm 0.3$ & $50.0 \pm 2.7$ & $47.0 \pm 6.8$ & $75.9 \pm 10.2$ & $61.9 \pm 0.2$ & $42.3 \pm 5.4$ & $40.6 \pm 2.6$ \\
\hline
\end{tabular}

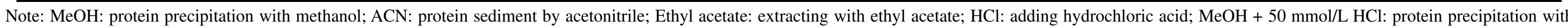
methanol under acidification. 


\section{Method validation}

specificity

Under the developed chromatographic and mass conditions, the sample chromatograms of blank plasma, the standard sample solution, the drug-containing plasma samples after oral administration of GZD, and the solution of GZD were presented in Fig. 2. The method had high specificity and could be used for the qualitative determination of these compounds in plasma.

\section{Linearity, $L L O Q$, and $L L O D$}

The results showed that the 10 compounds had good linearity in the corresponding concentration range. The regression equation, linear range and correlation coefficient ( $r$, LLOQ, and lower limit of detection (LLOD) of each compound were listed in Table 3.

\section{Precision and accuracy}

The results of precision and accuracy at the three different concentration levels were presented in Table 4. The intra- and inter-day RSD values were below $6.9 \%$ and $9.6 \%$ respectively, while the corresponding RE values ranged from $-10.8 \%$ to $5.3 \%$. All the assay values were within the acceptable criteria.

\section{Matrix effect and extraction recovery}

Average recoveries of investigated targets ranged from $85.4 \%$ to $113.9 \%$. The RSD values of all analytes were lower than $15.3 \%$, which indicated that there was no significant loss of the compounds among the process of the protein precipitation in the plasma samples. The matrix effect of each compound ranged from $85.4 \%$ to $116.8 \%$, indicating that there was no obvious matrix interference. The results showed that the method was accurate and acceptable. The data of extraction recovery and matrix effect were listed in Table 5.

\section{Stability}

After storage at $-80^{\circ} \mathrm{C}$ for 15 days and three freeze-thaw cycles, the stability RSD values of 10 compounds in plasma were all less than 9.9\% (Table 6). The results showed that the detected analytes were all satisfied with the criteria under all conditions, so the samples were stable during the test process. 


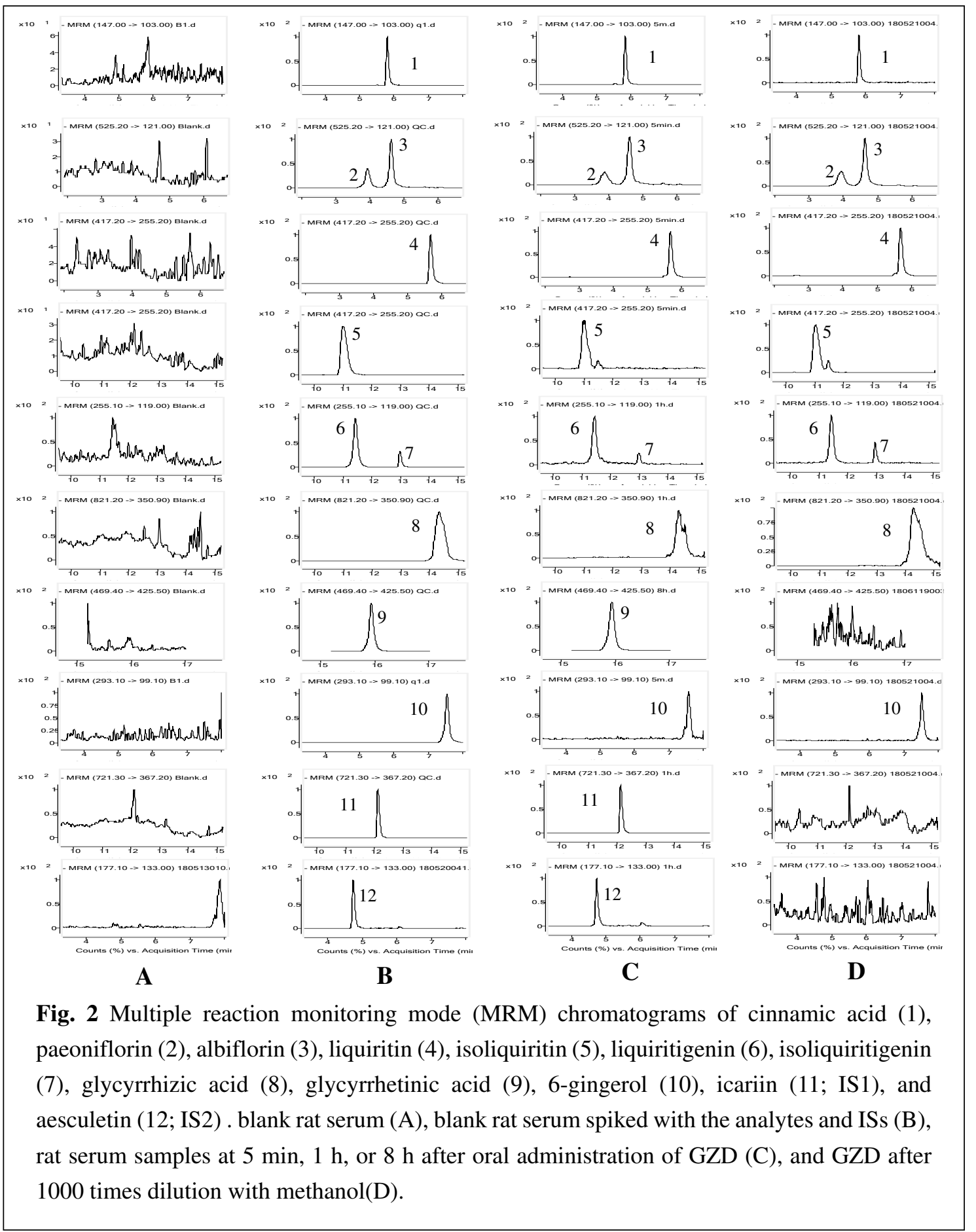


Table 3 Calibration curve, linear range, lower limit of detection (LLOD), and lower limit of quantification (LLOQ) of 10 analytes $(n=3)$

\begin{tabular}{llllcc}
\hline Analytes & Regression equation & $\begin{array}{l}\text { Liner range } \\
\left(\mathrm{ng} \cdot \mathrm{mL}^{-1}\right)\end{array}$ & $\boldsymbol{r}$ & $\begin{array}{c}\text { LLOQ } \\
\left(\mathrm{ng} \cdot \mathrm{mL}^{-1}\right)\end{array}$ & $\begin{array}{c}\text { LLOD } \\
\left(\mathrm{ng} \cdot \mathrm{mL}^{-1}\right)\end{array}$ \\
\hline Cinnamic acid & $\mathrm{y}=0.9385 \mathrm{x}+0.0344$ & $6-6120$ & 0.9995 & 6 & 2 \\
Paeoniflorin & $\mathrm{y}=0.3622 \mathrm{x}+0.0136$ & $6-1624$ & 0.9994 & 6 & 2 \\
Albiflorin & $\mathrm{y}=0.3895 \mathrm{x}-0.0395$ & $13-1635$ & 0.9996 & 13 & 3 \\
Liquiritin & $\mathrm{y}=2.5403 \mathrm{x}+0.0443$ & $1-1624$ & 0.9996 & 1 & 1 \\
Isoliquiritin & $\mathrm{y}=2.2788 \mathrm{x}+0.0393$ & $3-1624$ & 0.9997 & 3 & 1 \\
Liquiritigenin & $\mathrm{y}=1.8926 \mathrm{x}-0.0016$ & $4-1864$ & 0.9993 & 4 & 1 \\
Isoliquiritigenin & $\mathrm{y}=3.0726 \mathrm{x}+0.0336$ & $3-812$ & 0.9991 & 3 & 1 \\
Glycyrrhizic acid & $\mathrm{y}=0.4481 \mathrm{x}+0.0487$ & $7-1744$ & 0.9993 & 7 & 3 \\
Glycyrrhetinic acid & $\mathrm{y}=0.4370 \mathrm{x}+0.0427$ & $4-2090$ & 0.9993 & 4 & 2 \\
6-Gingerol & $\mathrm{y}=1.0994 \mathrm{x}+0.0392$ & $7-1784$ & 0.9983 & 7 & 3 \\
\hline
\end{tabular}

Table 4 Intra-day and inter-day precision and accuracy data of the 10 compounds spiked in rat plasma (mean $\pm \mathrm{SD} ; 3$ replicates per day for 3 days)

\begin{tabular}{|c|c|c|c|c|c|c|c|}
\hline \multirow[b]{2}{*}{ Analytes } & \multirow{2}{*}{$\begin{array}{l}\text { Spiked } \\
\text { conc. } \\
(\text { ng·mL } \\
-1)\end{array}$} & \multicolumn{3}{|c|}{ Intra-Day $(n=5)$} & \multicolumn{3}{|c|}{ Inter-Day $(n=5)$} \\
\hline & & $\begin{array}{l}\text { Conc. } \\
\left(\mathbf{n g} \cdot \mathbf{m L}^{-1}\right)\end{array}$ & $\begin{array}{l}\text { RSD } \\
\%\end{array}$ & $\begin{array}{l}\text { RE } \\
\%\end{array}$ & $\begin{array}{l}\text { Conc. } \\
\left(\mathbf{n g} \cdot \mathbf{m L}^{-1}\right)\end{array}$ & $\begin{array}{l}\text { RSD } \\
\%\end{array}$ & $\begin{array}{l}\text { RE } \\
\%\end{array}$ \\
\hline \multirow{3}{*}{ Cinnamic acid } & 51 & $51.3 \pm 0.9$ & 2.0 & 0.6 & $47.8 \pm 4.1$ & 8.8 & -6.2 \\
\hline & 204 & $209.4 \pm 9.6$ & 5.1 & 2.7 & $203.0 \pm 17.2$ & 8.8 & -0.5 \\
\hline & 812 & $818.6 \pm 31.3$ & 4.3 & 0.3 & $815.6 \pm 22.7$ & 2.9 & -0.1 \\
\hline \multirow{3}{*}{ Paeoniflorin } & 51 & $50.8 \pm 1.9$ & 4.2 & 0.1 & $53.1 \pm 3.5$ & 6.8 & 4.6 \\
\hline & 203 & $202.9 \pm 10.0$ & 5.5 & -0.1 & $200.1 \pm 13.1$ & 6.8 & -1.4 \\
\hline & 812 & $807.6 \pm 38.5$ & 5.3 & -0.6 & $812.6 \pm 21.4$ & 2.7 & 0.1 \\
\hline \multirow{3}{*}{ Albiflorin } & 55 & $54.5 \pm 1.1$ & 2.2 & -0.1 & $51.9 \pm 4.5$ & 8.9 & -4.8 \\
\hline & 218 & $218.0 \pm 7.6$ & 3.9 & 0.0 & $220.6 \pm 12.0$ & 5.6 & 1.2 \\
\hline & 872 & $886.5 \pm 39.0$ & 4.9 & 1.7 & $870.6 \pm 20.4$ & 2.4 & -0.2 \\
\hline \multirow{3}{*}{ Liquiritin } & 51 & $53.2 \pm 2.4$ & 5.0 & 4.8 & $48.5 \pm 4.5$ & 9.6 & -4.5 \\
\hline & 203 & $198.2 \pm 6.4$ & 3.6 & -2.4 & $205.4 \pm 10.6$ & 5.3 & 1.2 \\
\hline & 812 & $812.9 \pm 22.1$ & 3.0 & 0.1 & $811.5 \pm 21.1$ & 2.7 & -0.1 \\
\hline \multirow{3}{*}{ Isoliquiritin } & 51 & $49.4 \pm 1.0$ & 2.3 & -2.6 & $46.7 \pm 3.9$ & 8.7 & -8.1 \\
\hline & 203 & $208.1 \pm 12.8$ & 6.9 & 2.5 & $208.1 \pm 17.1$ & 8.5 & 2.5 \\
\hline & 812 & $810.6 \pm 44.0$ & 6.1 & -0.2 & $802.7 \pm 40.5$ & 5.2 & -1.2 \\
\hline \multirow{3}{*}{ Liquiritigenin } & 58 & $55.6 \pm 2.0$ & 4.0 & -4.5 & $54.3 \pm 4.3$ & 8.2 & -6.7 \\
\hline & 233 & $232.5 \pm 5.6$ & 2.7 & -0.2 & $242.8 \pm 20.5$ & 8.7 & 4.2 \\
\hline & 932 & $850.1 \pm 52.5$ & 6.9 & -8.8 & $888.0 \pm 41.2$ & 4.8 & -4.7 \\
\hline \multirow{3}{*}{ Isoliquiritigenin } & 51 & $47.3 \pm 1.7$ & 4.1 & -6.9 & $45.3 \pm 3.8$ & 8.8 & -10.8 \\
\hline & 203 & $207.4 \pm 9.5$ & 5.1 & 2.2 & $207.5 \pm 15.7$ & 7.8 & 2.2 \\
\hline & 812 & $793.3 \pm 44.8$ & 6.3 & -2.3 & $794.9 \pm 32.1$ & 4.2 & -2.1 \\
\hline \multirow{3}{*}{ Glycyrrhizic acid } & 55 & $57.4 \pm 1.3$ & 2.6 & 5.3 & $52.0 \pm 3.8$ & 7.5 & -4.7 \\
\hline & 218 & $213.3 \pm 7.2$ & 3.8 & -2.2 & $223.2 \pm 13.9$ & 6.4 & 2.4 \\
\hline & 872 & $808.5 \pm 22.5$ & 3.1 & -7.3 & $876.2 \pm 33.0$ & 3.9 & 0.5 \\
\hline \multirow{3}{*}{$\begin{array}{l}\text { Glycyrrhetinic } \\
\text { acid }\end{array}$} & 52 & $49.5 \pm 1.0$ & 2.2 & -5.3 & $47.5 \pm 2.5$ & 5.4 & -9.1 \\
\hline & 209 & $216.3 \pm 11.1$ & 5.8 & 3.5 & $216.2 \pm 15.6$ & 7.5 & 3.4 \\
\hline & 836 & $834.4 \pm 35.3$ & 4.7 & -0.2 & $830.2 \pm 29.0$ & 3.6 & -0.7 \\
\hline \multirow{3}{*}{ 6-Gingerol } & 56 & $51.6 \pm 2.1$ & 4.6 & -7.5 & $53.7 \pm 3.8$ & 7.3 & -3.6 \\
\hline & 223 & $201.4 \pm 1.6$ & 0.9 & -9.7 & $220.7 \pm 18.1$ & 8.5 & -1.0 \\
\hline & 892 & $833.8 \pm 35.6$ & 4.8 & -6.5 & $890.3 \pm 20.1$ & 2.3 & -0.2 \\
\hline
\end{tabular}

Note: RSD: relative standard deviation; RE: refractive error. 
Table 5 Extraction recovery and matrix effect data of the 10 compounds in rat plasma ( $n$ =5)

\begin{tabular}{|c|c|c|c|c|c|}
\hline \multirow{2}{*}{ Analytes } & \multirow{2}{*}{$\begin{array}{l}\text { Spiked } \\
\text { conc. } \\
\left(\mathbf{n g} \cdot \mathbf{m L}^{-1}\right)\end{array}$} & \multicolumn{2}{|c|}{ Extraction Recovery (\%) } & \multicolumn{2}{|c|}{ Matrix Effect $(\%)$} \\
\hline & & Mean & RSD & Mean & RSD \\
\hline \multirow{3}{*}{ Cinnamic acid } & 51 & 97.9 & 15.3 & 96.1 & 8.9 \\
\hline & 204 & 96.3 & 8.7 & 103.3 & 8.5 \\
\hline & 812 & 110.9 & 4.8 & 90.3 & 6.0 \\
\hline \multirow{3}{*}{ Paeoniflorin } & 51 & 94.1 & 5.8 & 111.0 & 8.3 \\
\hline & 203 & 99.4 & 2.4 & 96.8 & 2.2 \\
\hline & 812 & 108.2 & 7.1 & 92.6 & 4.8 \\
\hline \multirow{3}{*}{ Albiflorin } & 55 & 95.9 & 9.8 & 102.4 & 10.5 \\
\hline & 218 & 104.8 & 5.8 & 85.4 & 9.4 \\
\hline & 872 & 113.9 & 9.4 & 88.7 & 7.5 \\
\hline \multirow{3}{*}{ Liquiritin } & 51 & 106.3 & 6.2 & 100.1 & 6.2 \\
\hline & 203 & 91.0 & 4.0 & 104.5 & 4.0 \\
\hline & 812 & 91.8 & 5.3 & 116.8 & 9.5 \\
\hline \multirow{3}{*}{ Isoliquiritin } & 51 & 87.6 & 11.3 & 106.4 & 3.9 \\
\hline & 203 & 110.3 & 6.6 & 93.0 & 4.0 \\
\hline & 812 & 102.0 & 5.4 & 98.1 & 2.5 \\
\hline \multirow{3}{*}{ Liquiritigenin } & 58 & 97.1 & 3.9 & 97.8 & 2.6 \\
\hline & 233 & 101.8 & 2.3 & 97.8 & 2.0 \\
\hline & 932 & 105.5 & 7.3 & 103.3 & 6.5 \\
\hline \multirow{3}{*}{ Isoliquiritigenin } & 51 & 92.6 & 5.9 & 108.6 & 4.6 \\
\hline & 203 & 105.2 & 4.9 & 105.8 & 3.2 \\
\hline & 812 & 97.2 & 2.7 & 113.7 & 7.4 \\
\hline \multirow{3}{*}{ Glycyrrhizic acid } & 55 & 100.7 & 5.4 & 114.8 & 9.0 \\
\hline & 218 & 89.1 & 5.9 & 105.7 & 4.3 \\
\hline & 872 & 87.5 & 8.8 & 94.7 & 12.7 \\
\hline \multirow{3}{*}{ Glycyrrhetinic acid } & 52 & 88.2 & 8.3 & 94.0 & 7.2 \\
\hline & 209 & 88.1 & 8.2 & 105.4 & 4.7 \\
\hline & 836 & 94.5 & 3.9 & 106.2 & 4.4 \\
\hline \multirow{3}{*}{ 6-Gingerol } & 56 & 85.4 & 7.9 & 111.3 & 6.7 \\
\hline & 223 & 88.0 & 6.9 & 88.6 & 9.1 \\
\hline & 892 & 109.5 & 4.3 & 86.5 & 8.0 \\
\hline
\end{tabular}

Table 6 Stability of 10 compounds under various conditions $(n=5)$.

\begin{tabular}{|c|c|c|c|c|c|c|c|}
\hline \multirow{2}{*}{ Analytes } & \multirow{2}{*}{$\begin{array}{l}\text { Spiked } \\
\text { conc. } \\
\left(\text { ng } \cdot \mathbf{m L}^{-1}\right)\end{array}$} & \multicolumn{2}{|c|}{$\begin{array}{c}12 \mathrm{~h} \text { at } 4^{\circ} \mathrm{C} \\
(\%)\end{array}$} & \multicolumn{2}{|c|}{$\begin{array}{c}\text { Three Freeze- } \\
\text { Thaw Cycles (\%) }\end{array}$} & \multicolumn{2}{|c|}{$\begin{array}{c}15 \text { Days } \\
\text { at }-80^{\circ} \mathrm{C}(\%)\end{array}$} \\
\hline & & Mean & RSD & Mean & RSD & Mean & RSD \\
\hline \multirow{3}{*}{ Cinnamic acid } & 52 & 97.5 & 8.4 & 92.1 & 4.0 & 101.1 & 8.4 \\
\hline & 209 & 111.2 & 5.1 & 102.5 & 6.3 & 109.4 & 8.0 \\
\hline & 836 & 111.4 & 6.1 & 99.9 & 1.9 & 99.5 & 4.6 \\
\hline \multirow{3}{*}{ Paeoniflorin } & 51 & 88.8 & 6.2 & 89.6 & 4.0 & 104.6 & 7.7 \\
\hline & 203 & 85.6 & 6.3 & 103.3 & 4.4 & 97.5 & 3.1 \\
\hline & 812 & 99.5 & 8.5 & 99.9 & 2.7 & 100.1 & 4.7 \\
\hline \multirow{3}{*}{ Albiflorin } & 55 & 100.7 & 9.9 & 96.2 & 6.5 & 90.6 & 1.9 \\
\hline & 218 & 90.1 & 8.5 & 100.5 & 4.4 & 104.2 & 8.3 \\
\hline & 872 & 102.6 & 6.8 & 100.0 & 1.3 & 101.2 & 4.7 \\
\hline \multirow{3}{*}{ Liquiritin } & 51 & 92.2 & 5.8 & 89.7 & 2.7 & 93.3 & 0.6 \\
\hline & 203 & 103.6 & 5.6 & 103.2 & 5.2 & 106.7 & 5.1 \\
\hline & 812 & 98.2 & 5.8 & 99.8 & 3.8 & 99.7 & 2.0 \\
\hline Isoliquiritin & 51 & 109.1 & 4.6 & 87.2 & 1.6 & 104.8 & 5.2 \\
\hline
\end{tabular}




\begin{tabular}{llllllll} 
& 203 & 90.9 & 5.4 & 104.0 & 4.5 & 95.8 & 7.9 \\
& 812 & 96.1 & 7.6 & 99.8 & 4.0 & 100.9 & 1.3 \\
\hline \multirow{3}{*}{ Liquiritigenin } & 58 & 95.4 & 9.5 & 89.1 & 2.6 & 95.6 & 7.9 \\
& 233 & 102.9 & 3.4 & 103.4 & 6.1 & 109.4 & 6.3 \\
& 932 & 108.3 & 7.3 & 99.8 & 4.5 & 99.3 & 2.1 \\
\hline \multirow{3}{*}{ Isoliquiritigenin } & 51 & 109.5 & 8.8 & 90.7 & 2.5 & 97.5 & 8.8 \\
& 203 & 90.2 & 5.2 & 102.9 & 5.6 & 101.5 & 2.3 \\
& 812 & 97.5 & 6.7 & 99.9 & 5.1 & 99.9 & 4.0 \\
\hline \multirow{3}{*}{ Glycyrrhizic acid } & 54.555 & 99.3 & 5.8 & 89.8 & 4.4 & 98.0 & 1.8 \\
& 218 & 101.0 & 1.8 & 103.2 & 2.1 & 104.7 & 4.3 \\
& 872 & 102.9 & 5.7 & 99.9 & 5.8 & 99.8 & 1.6 \\
\hline \multirow{3}{*}{ Glycyrrhetinic acid } & 52 & 100.4 & 8.5 & 90.5 & 5.1 & 103.6 & 7.5 \\
& 209 & 99.5 & 6.8 & 105.2 & 6.2 & 108.3 & 3.7 \\
& 836 & 105.6 & 6.4 & 99.7 & 2.9 & 99.6 & 4.2 \\
\hline \multirow{3}{*}{ 6-Gingerol } & 56 & 98.8 & 5.1 & 87.5 & 4.6 & 97.5 & 7.0 \\
& 223 & 99.3 & 4.6 & 103.9 & 4.3 & 100.1 & 4.1 \\
& 892 & 103.5 & 7.0 & 99.8 & 3.5 & 100.0 & 0.3 \\
\hline
\end{tabular}

Note: Mean: measured concentration; RSD: relative standard deviation.

\section{Pharmacokinetic results}

The validated HPLC-MS/MS methods were applied to the pharmacokinetic study of the 10 compounds in rat plasma after oral administration and intravenous injection of GZD. The pharmacokinetic parameters were calculated by Phoenix Winnonlin 8.1 software (Certara, USA). Mean blood concentration-time curves (C-T) of the 10 compounds taken orally were displayed in Fig. 3, and the main pharmacokinetic parameters were listed in Table 7. Simultaneously, the pharmacokinetic results of the compounds administered intravenously were mainly shown in Fig. 4 and Table 8.

After oral administration of GZD, 10 compounds were detected in plasma. Except for glycyrrhetinic acid (metabolite), the other compounds reached the maximum blood concentration quickly, whose $\mathrm{T}_{\max }$ was about 0.1-0.2 min. This pharmacokinetics phenomenon coincided with the characteristic of GZD as the Jiebiao formula (TCM term), which took effect quickly. All the AUC and $\mathrm{C}_{\max }$ values of those 10 compounds at different doses were positively correlated with the dose, and $T_{1 / 2}$ was independent with the dose, indicating that the compounds were in line with the firstorder kinetic process in vivo.

A total of 9 compounds were detected after intravenous injection of GZD. The plasma concentration-time curve of these compounds declines rapidly at the beginning, and then decreased slowly, indicating that the plasma concentration-time curves were double exponential function curves. Initially, these compounds did not reach the dynamic balance (the effects of distribution and elimination combined together). After a period of time, only the elimination process existed in vivo. The metabolites, glycyrrhetinic acid, was detected in plasma. However, it could not be fitted with winnonlin software due to its concentration was too low.The V_F values of paenoiflorin, liquiritigenin, and isoliquiritigenin were higher than those of other compounds, which indicated they had broad distribution in tissues.Glycyrrhizic acid had the longest $\mathrm{T}_{1 / 2}$ and MRT. The compounds eliminated slowly in vivo. 


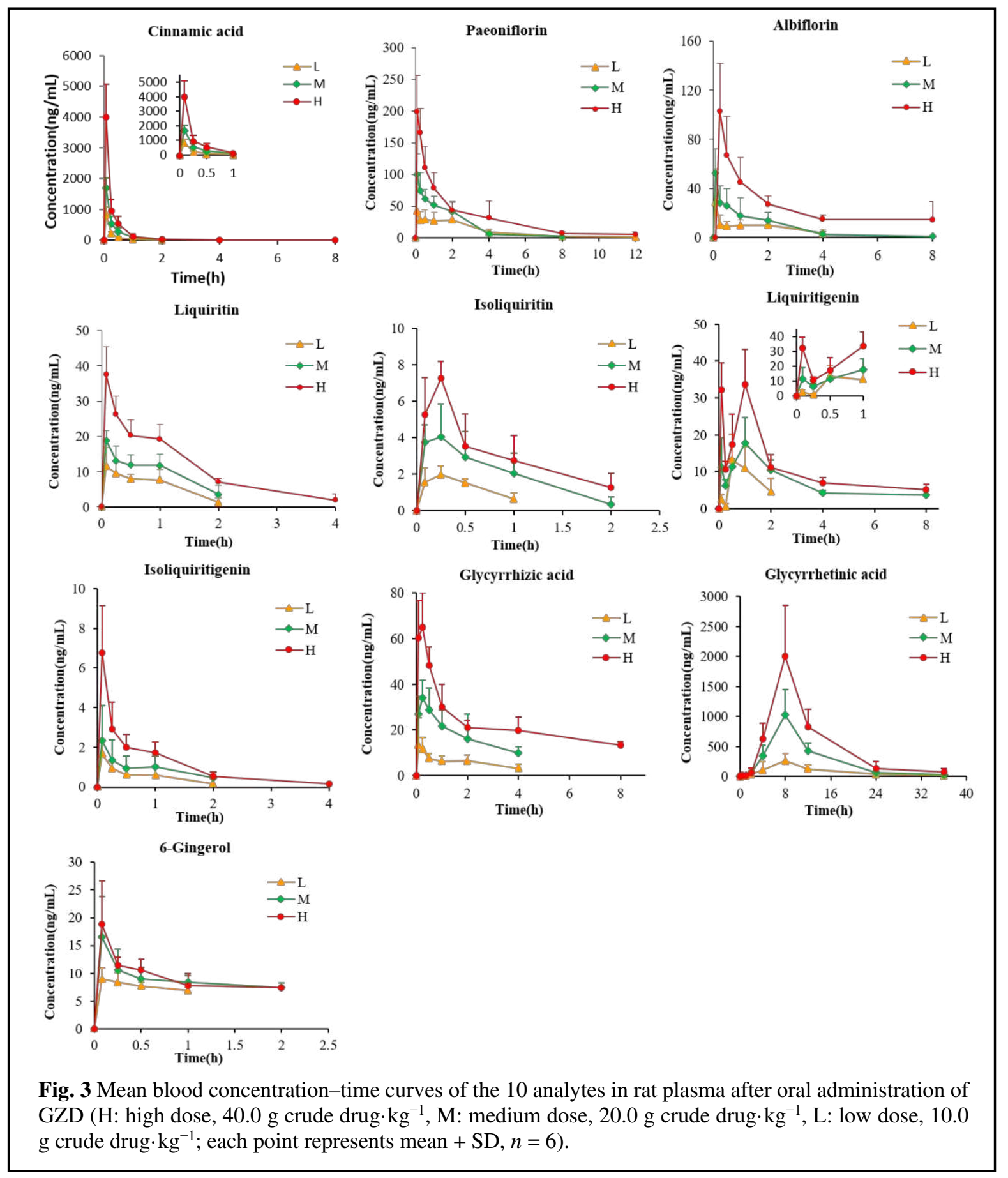




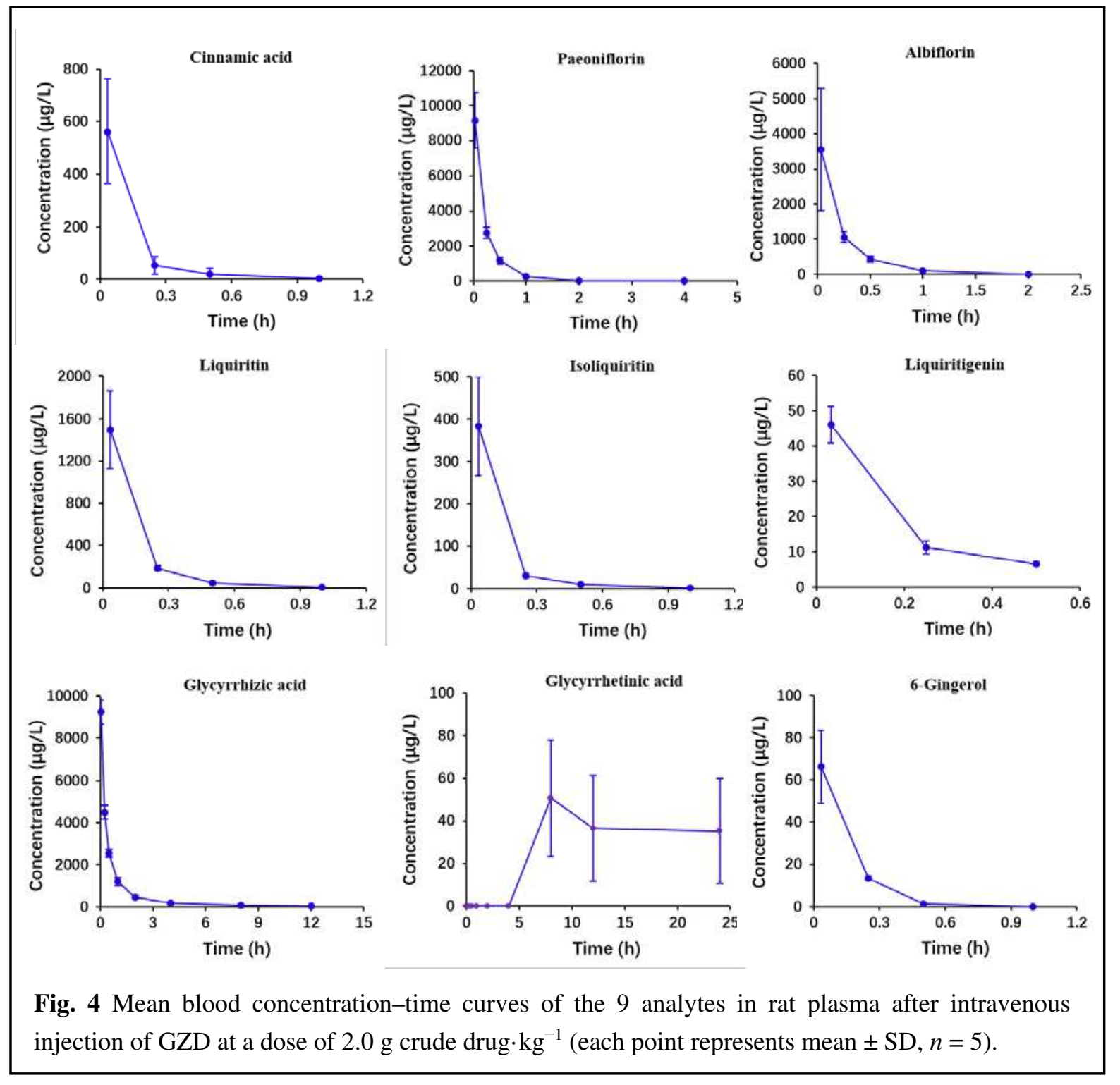


Table 7 Pharmacokinetic parameters of 10 compounds in rat plasma after oral administration of GZD in three doses (H: high dose, $40.0 \mathrm{~g}$ crude drug $\cdot \mathrm{kg}^{-1}$, M: medium dose, $20.0 \mathrm{~g}$ crude drug $\cdot \mathrm{kg}^{-1}$, L: low dose, $10.0 \mathrm{~g}$ crude drug $\cdot \mathrm{kg}^{-1} ; n=6$ )

\begin{tabular}{|c|c|c|c|c|c|c|c|c|}
\hline Analytes & Dose & $\begin{array}{l}\mathbf{A U} \mathbf{C}_{0-\infty} \\
\left(\mathbf{h} \cdot \boldsymbol{\mu g} \cdot \mathbf{L}^{-1}\right)\end{array}$ & $\begin{array}{l}\mathbf{T}_{1 / 2} \\
(\mathbf{h}) \\
\end{array}$ & $\begin{array}{l}\text { Tmax } \\
\text { (h) }\end{array}$ & $\begin{array}{l}\operatorname{Cmax} \\
\left(\mu g \cdot \mathbf{L}^{-1}\right)\end{array}$ & $\begin{array}{l}\text { Vz_F } \\
\left(\mathbf{L} \cdot \mathbf{k g}^{-\mathbf{1}}\right) \\
\end{array}$ & $\begin{array}{l}\text { Cl_F } \\
\left(\mathbf{L} \cdot \mathbf{h}^{-1} \cdot \mathbf{k g}^{-1}\right)\end{array}$ & $\begin{array}{l}\text { MRT }_{0-t} \\
\text { (h) }\end{array}$ \\
\hline \multirow{3}{*}{ Cinnamic acid } & $\mathrm{H}$ & $1059.3 \pm 221.1$ & $0.5 \pm 0.1$ & $0.1 \pm 0.0$ & $4027.6 \pm 1046.7$ & $5.2 \pm 3.5$ & $3.3 \pm 0.6$ & $0.5 \pm 0.1$ \\
\hline & M & $396.5 \pm 124.3$ & $0.3 \pm 0.1$ & $0.1 \pm 0.1$ & $1004.0 \pm 253.0$ & $1.9 \pm 0.8$ & $4.8 \pm 2.0$ & $0.4 \pm 0.1$ \\
\hline & $\mathrm{L}$ & $186.4 \pm 63.8$ & $0.2 \pm 0.0$ & $0.1 \pm 0.0$ & $831.3 \pm 250.1$ & $1.3 \pm 0.4$ & $5.1 \pm 2.0$ & $0.2 \pm 0.1$ \\
\hline \multirow{3}{*}{ Paeoniflorin } & $\mathrm{H}$ & $353.1 \pm 123.8$ & $2.1 \pm 0.4$ & $0.1 \pm 0.1$ & $221.3 \pm 64.1$ & $952.8 \pm 338.6$ & $319.8 \pm 93.3$ & $2.6 \pm 0.5$ \\
\hline & M & $175.2 \pm 46.7$ & $1.8 \pm 0.4$ & $0.1 \pm 0.0$ & $100.7 \pm 31.9$ & $618.2 \pm 170.1$ & $316.5 \pm 94.3$ & $2.0 \pm 0.2$ \\
\hline & $\mathrm{L}$ & $46.8 \pm 8.7$ & $2.8 \pm 3.4$ & $0.5 \pm 0.7$ & $31.0 \pm 25.1$ & $858.5 \pm 599.4$ & $519.8 \pm 60.2$ & $4.2 \pm 4.8$ \\
\hline \multirow{3}{*}{ Albiflorin } & $\mathrm{H}$ & $138.9 \pm 55.9$ & $1.8 \pm 0.3$ & $0.1 \pm 0.1$ & $107.1 \pm 42.1$ & $992.9 \pm 335.6$ & $394.8 \pm 159.7$ & $2.3 \pm 0.6$ \\
\hline & M & $67.1 \pm 33.1$ & $1.5 \pm 0.4$ & $0.1 \pm 0.0$ & $52.6 \pm 19.3$ & $886.5 \pm 381.0$ & $426.6 \pm 179.3$ & $1.9 \pm 0.5$ \\
\hline & $\mathrm{L}$ & $46.8 \pm 8.7$ & $1.6 \pm 1.2$ & $0.5 \pm 0.7$ & $31.0 \pm 25.1$ & $1037.8 \pm 1304.7$ & $262.4 \pm 51.4$ & $2.2 \pm 1.1$ \\
\hline \multirow{3}{*}{ Liquiritin } & $\mathrm{H}$ & $47.1 \pm 11.3$ & $0.9 \pm 0.3$ & $0.1 \pm 0.0$ & $37.6 \pm 7.9$ & $2834.8 \pm 574.8$ & $2417.7 \pm 450.2$ & $1.3 \pm 0.3$ \\
\hline & M & $27.6 \pm 12.8$ & $0.9 \pm 0.5$ & $0.1 \pm 0.1$ & $19.2 \pm 2.3$ & $2554.0 \pm 656.6$ & $2277.9 \pm 794.6$ & $1.3 \pm 0.6$ \\
\hline & $\mathrm{L}$ & $12.3 \pm 1.7$ & $0.5 \pm 0.1$ & $0.5 \pm 0.4$ & $13.7 \pm 4.1$ & $1626.9 \pm 280.2$ & $2261.8 \pm 335.8$ & $0.8 \pm 0.1$ \\
\hline \multirow{3}{*}{ Isoliquiritin } & $\mathrm{H}$ & $8.0 \pm 3.7$ & $0.9 \pm 0.5$ & $0.2 \pm 0.1$ & $7.8 \pm 0.9$ & $2984.1 \pm 1349.0$ & $2491.4 \pm 898.8$ & $1.3 \pm 0.6$ \\
\hline & M & $5.0 \pm 1.8$ & $1.2 \pm 1.6$ & $0.1 \pm 0.1$ & $4.7 \pm 1.3$ & $1976.4 \pm 1291.6$ & $1779.6 \pm 940.7$ & $1.8 \pm 2.3$ \\
\hline & $\mathrm{L}$ & $1.5 \pm 0.1$ & $1.1 \pm 0.8$ & $0.2 \pm 0.2$ & $2.2 \pm 0.4$ & $1084.1 \pm 70.7$ & $2584.6 \pm 212.1$ & $1.2 \pm 1.0$ \\
\hline \multirow{3}{*}{ Liquiritigenin } & $\mathrm{H}$ & $102.0 \pm 39.2$ & $3.3 \pm 1.9$ & $0.6 \pm 0.5$ & $42.3 \pm 17.4$ & $192.8 \pm 57.9$ & $36.7 \pm 8.5$ & $4.2 \pm 2.3$ \\
\hline & M & $72.3 \pm 0.3$ & $4.1 \pm 0.4$ & $0.8 \pm 0.4$ & $19.9 \pm 7.6$ & $170.9 \pm 16.8$ & $29.1 \pm 0.1$ & $5.8 \pm 0.8$ \\
\hline & $\mathrm{L}$ & $16.1 \pm 9.7$ & $3.9 \pm 0.1$ & $0.8 \pm 0.3$ & $13.4 \pm 6.8$ & $198.2 \pm 46.1$ & $43.0 \pm 3.9$ & $4.3 \pm 1.7$ \\
\hline \multirow{3}{*}{ Isoliquiritigenin } & $\mathrm{H}$ & $4.5 \pm 0.9$ & $0.9 \pm 0.2$ & $0.1 \pm 0.0$ & $6.8 \pm 3.4$ & $299.7 \pm 98.4$ & $227.7 \pm 51.8$ & $1.2 \pm 0.3$ \\
\hline & M & $2.9 \pm 1.1$ & $1.1 \pm 0.8$ & $0.1 \pm 0.1$ & $2.7 \pm 1.6$ & $356.2 \pm 101.2$ & $211.1 \pm 95.3$ & $2.0 \pm 1.6$ \\
\hline & $\mathrm{L}$ & $1.1 \pm 0.1$ & $0.6 \pm 0.3$ & $0.2 \pm 0.4$ & $1.8 \pm 0.7$ & $196.4 \pm 120.1$ & $229.0 \pm 31.6$ & $0.8 \pm 0.4$ \\
\hline \multirow{3}{*}{ Glycyrrhizic acid } & $\mathrm{H}$ & $174.8 \pm 36.9$ & $3.0 \pm 0.3$ & $0.2 \pm 0.2$ & $73.6 \pm 20.9$ & $530.9 \pm 116.0$ & $120.9 \pm 24.2$ & $4.2 \pm 0.4$ \\
\hline & M & $109.9 \pm 43.0$ & $3.2 \pm 1.0$ & $0.5 \pm 0.8$ & $44.7 \pm 25.6$ & $334.2 \pm 173.9$ & $107.2 \pm 49.8$ & $3.2 \pm 1.5$ \\
\hline & $\mathrm{L}$ & $54.8 \pm 18.5$ & $3.8 \pm 2.1$ & $0.5 \pm 0.8$ & $15.3 \pm 10.0$ & $540.7 \pm 194.0$ & $105.1 \pm 47.9$ & $5.8 \pm 3.1$ \\
\hline \multirow{3}{*}{$\begin{array}{l}\text { Glycyrrhetinic } \\
\text { acid }\end{array}$} & $\mathrm{H}$ & $18958.4 \pm 6548.3$ & $6.6 \pm 2.3$ & $8.0 \pm 0.0$ & $2000.2 \pm 854$ & $10.5 \pm 3.2$ & $1.2 \pm 0.4$ & $12 \pm 2.6$ \\
\hline & M & $9781.7 \pm 2315.9$ & $6.4 \pm 1.9$ & $8.0 \pm 0.0$ & $1021.9 \pm 425.1$ & $10.3 \pm 4.7$ & $1.1 \pm 0.3$ & $11.6 \pm 0.8$ \\
\hline & $\mathrm{L}$ & $3275.4 \pm 993.6$ & $6.2 \pm 2.9$ & $7.3 \pm 3.0$ & $283.8 \pm 115.9$ & $14.1 \pm 6.4$ & $1.7 \pm 0.4$ & $12.3 \pm 4.7$ \\
\hline
\end{tabular}




\begin{tabular}{lllllllll}
\hline \multirow{3}{*}{ 6-Gingerol } & $\mathrm{H}$ & $76.0 \pm 64.3$ & $3.2 \pm 1.9$ & $0.2 \pm 0.2$ & $24.2 \pm 10.1$ & $155.6 \pm 14.8$ & $43.7 \pm 24.2$ & $4.5 \pm 2.7$ \\
& $\mathrm{M}$ & $45.0 \pm 5.0$ & $2.5 \pm 0.9$ & $0.6 \pm 0.7$ & $17.5 \pm 16.9$ & $67.8 \pm 17.3$ & $19.5 \pm 2.2$ & $3.4 \pm 1.5$ \\
& $\mathrm{~L}$ & $28.1 \pm 0.0$ & $2.9 \pm 1.1$ & $0.1 \pm 0.1$ & $9.9 \pm 1.5$ & $151.2 \pm 146.1$ & $31.5 \pm 22.6$ & $3.6 \pm 0.6$ \\
\hline
\end{tabular}

Table 8 Pharmacokinetic parameters of 10 compounds in rat plasma after intravenous injection of GZD at a dose of $2.0 \mathrm{~g}$ crude drug. $\operatorname{kg}^{-1}(\mathbf{n}=6)$

\begin{tabular}{|c|c|c|c|c|c|c|}
\hline Analytes & $\begin{array}{l}C_{0} \\
\left(\mu g \cdot L^{-1}\right)\end{array}$ & $\begin{array}{l}A \mathbf{U} C_{0-\infty} \\
\left(\mathbf{h} \cdot \boldsymbol{\mu g} \cdot \mathbf{L}^{-1}\right)\end{array}$ & $\begin{array}{l}\mathbf{T}_{1 / 2} \\
(\mathbf{h})\end{array}$ & $\begin{array}{l}\text { VsS } \\
\left(\mathbf{L} \cdot \mathbf{k g}^{-1}\right) \\
\end{array}$ & $\begin{array}{l}\text { Cl_F } \\
\left(\mathbf{L} \cdot \mathbf{h}^{-1} \cdot \mathbf{k g}^{-1}\right)\end{array}$ & $\begin{array}{l}\text { MRT }_{\text {0-t }} \\
\text { (h) }\end{array}$ \\
\hline Cinnamic acid & $106.4 \pm 40.3$ & $821.6 \pm 310.7$ & $0.2 \pm 0.0$ & $0.2 \pm 0.0$ & $1.8 \pm 0.9$ & $0.3 \pm 0.0$ \\
\hline Paeoniflorin & $2671.4 \pm 354$ & $11038.6 \pm 2175.8$ & $0.5 \pm 0.2$ & $0.6 \pm 0.2$ & $2.0 \pm 0.3$ & $0.6 \pm 0.0$ \\
\hline Albiflorin & $987.3 \pm 226.3$ & $4386.1 \pm 2358.1$ & $0.2 \pm 0.0$ & $0.7 \pm 0.4$ & $2.6 \pm 0.7$ & $0.2 \pm 0.1$ \\
\hline Liquiritin & $2072.0 \pm 588.0$ & $285.2 \pm 59.0$ & $0.2 \pm 0.0$ & $2.2 \pm 0.5$ & $21.2 \pm 4.4$ & $0.3 \pm 0.0$ \\
\hline Isoliquiritin & $68.1 \pm 18.8$ & $570.8 \pm 200.6$ & $0.1 \pm 0.1$ & $1.1 \pm 0.5$ & $12.8 \pm 4.2$ & $0.1 \pm 0.0$ \\
\hline Liquiritigenin & $11.8 \pm 1.1$ & $57.3 \pm 7.0$ & $0.1 \pm 0.0$ & $0.7 \pm 0.1$ & $4.1 \pm 0.4$ & $0.2 \pm 0.0$ \\
\hline Glycyrrhizic acid & $5802.2 \pm 458.7$ & $10327.2 \pm 791.6$ & $2.3 \pm 0.6$ & $0.3 \pm 0.0$ & $0.2 \pm 0.0$ & $2.5 \pm 0.3$ \\
\hline 6-Gingerol & $12.9 \pm 2.6$ & $84.4 \pm 25.4$ & $0.1 \pm 0.0$ & $0.6 \pm 0.2$ & $6.7 \pm 1.3$ & $0.1 \pm 0.0$ \\
\hline
\end{tabular}




\section{Discussion}

The validated HPLC-MS/MS method was successfully applied to determination of 10 compounds, including 9 prototype compounds and 1 metabolite in rat plasma after oral administration and intravenous injection. Besides, we also studied the bioavailability of all measured substances in plasma based on the pharmacokinetics results to explore the absorption and utilization of GZD in vivo.

Ramulus Cinnamomi was the sovereign drug in GZD. The AUC of cinnamic acid(i.g.) were bigger than other prototypic compounds, while the content of cinnamic acid in decoction was much lower. It meant that cinnamic acid was more easily absorbed into blood.

Paeoniae Radix Alba was the assistant drug in GZD. Several papers had reported that there was a mutual transformation between paeoniflorin and albiflorin[26]. The ratios of AUC and $\mathrm{C}_{\max }$ of paeoniflorin and albiflorin were basically the same as the ratios of content in decoction (2:1) after oral or intravenous administration. This indicated that the two compounds might not be transferred into each other in vivo.

Radix Glycyrrhizae was the envoy drug in GZD. In this study, 6 compounds were detected in rat plasma after oral administration. The content of liquiritin was higher in GZD than that of other compounds, but its $\mathrm{C}_{\max }$ and AUC values were low, indicating that it was not easily absorbed into the body. The $\mathrm{C}_{\max }$ and $\mathrm{AUC}$ values of liquiritigenin were about twice that of liquiritin in vivo, but the content of liquiritigenin was much lower in GZD (1:100). The result suggested that liquiritin could be converted into liquiritigenin in vivo. Moreover, the C-T diagram of liquiritigenin showed a double peak, which might due to the transformation of liquiritin in the intestinal tract. The AUC ratio of liquiritin to liquiritigenin in the plasma during intravenous administration was similar to the ratio of their content in GZD, indicating that liquiritin could not be transformed into liquiritigenin by liver. Combining the results of oral administration, it could be speculated that the transformation process mainly occurred in intestines.Similar to liquiritin, glycyrrhizic acid had high content in GZD, but the values of $\mathrm{C}_{\max }$ and AUC were low after oral administration, indicating that it was not easily absorbed. The $\mathrm{C}_{\max }$ and AUC of glycyrrhetinic acid were about 60-100 times than that of glycyrrhizic acid, which shown that glycyrrhetinic acid were more easily absorbed into the body than glycyrrhizic acid. The $\mathrm{T}_{\max }$ of glycyrrhetinic acid was about $8 \mathrm{~h}$, indicating that the speed of conversion was slow. After intravenous injection, the AUC ratio of glycyrrhetinic acid to glycyrrhizic acid was significantly lower than the ratio of the two compounds after oral administration, suggesting that the liver could not convert glycyrrhizic acid into glycyrrhetinic acid effectively. We speculated that after GZD injected into blood, only a small part of glycyrrhizic acid was excreted into intestine by the bile of the liver, and metabolized into glycyrrhetinic acid by intestinal flora, then re-absorbed into the blood.

Rhizoma Zingiberis Recens were the supplementary drug in GZD. The Vz_F of 6-gingerol was relatively high , indicating that the compound was widely distributed in the body or accumulated in some tissues. Different from cinnamic acid, the $\mathrm{T}_{1 / 2}$ of 6-gingerol (about $3 \mathrm{~h}$ ) was greater than the $\mathrm{T}_{1 / 2}$ of cinnamic acid (about $0.5 \mathrm{~h}$ ), and the Cl_F and MRT values were higher than those of cinnamic acid, indicating that the elimination of 6-gingerol in the body was slow.

Based on the determination of the main compounds of GZD, we studied the pharmacokinetics of the main effective compounds of GZD through blood. These compounds are likely to be the basis of the pharmacodynamics of Guizhi Decoction. These studies will provide information to help clarify the biologically active ingredients and the mechanism of action of Chinese medicine prescriptions.

On the basis of compound chemical components research of GZD, we studied the major effective compounds observed in blood through the pharmacokinetics research, which were the mostly possible material basis of GZD[27]. Moreover, the research help to clarify the biologically active ingredients and mechanism of action in TCM prescriptions.

\section{Conclusions}

In this study, the HPLC-MS/MS analysis method was established for the quantification and determination of 10 compounds in GZD and 10 compounds (including 9 prototype compounds and 1 metabolites) in rat plasma. The results showed that the compounds in GZD were absorbed into the body with a first-order kinetics. At the dose of the study, the exposure of these compounds was 
proportional to the dose. The absorption was rapid, as the $\mathrm{t}_{\max }$ were about $0.1 \sim 0.2 \mathrm{~h}$.The results suggested that the intestinal digestion played an important role in the pharmacological effects of GZD. The active compounds of GZD might the metabolisms, rather than the prototypes. This process might affect the pharmacodynamics effects of GZD. We expected this results would help us to reveal the pharmacodynamic material basis of GZD and provide a reference for the rational use of GZD in the clinic.

\section{List of abbreviations}

GZD: Guizhi Decoction; TCM: traditional Chinese medicine; HPLC-MS/MS: high-performance liquid chromatography-tandem mass spectrometry; LLOQ: lower limit of quantification; LLOD: lower limit of detection; PK: pharmacokinetics; MRM: multiple reaction monitoring; IS: internal standard; RSD: relative standard deviation; RE: refractive error.

\section{Ethics approval and consent to participate}

The animal study was approved by Animal Care and Use Committee of the Institute of Basic Theory for Chinese Medicine, China Academy of Chinese Medical Sciences (Date: 25 March 2019; No.: SYXK (Jing) 2019-008).

\section{Consent to publish}

All authors consent to publication of this study in Chinese Medicine.

\section{Availability of data and materials}

The datasets used in this article are available from the corresponding author upon request.

\section{Competing interests}

The authors declare no conflict of interest.

\section{Funding}

This work was supported by the Youth Project of National Natural Science Foundation (No. 81403282) and the Natural Science Foundation of Beijing (No. 7133251).

\section{Authors'contributions}

H.G., Q. G., D.B. and X.W. designed the research and performed the HPLC experiment; L.Z., H.G. and Q. G. performed the animal experiment; H.G., Q. G., and M.B. analyzed the data and wrote the paper. All authors read and approved the final manuscript.

\section{Acknowledgements}

This study was financially supported by the Special Funding for Basic Scientific Research Business Expenses of Central Public Welfare Scientific Research Institutes (No. YZ202027).

\section{Author details}

${ }^{1}$ Institute of Basic Theory of Traditional Chinese Medicine, China Academy of Chinese Medical Sciences, Beijing, 100700, China

${ }^{2}$ College of pharmacy, Shaanxi University of Chinese Medicine, Xianyang, 712000, China

${ }^{3}$ Medical Experiment Center, China Academy of Traditional Chinese Medicine Sciences, Beijing, 100700, China

${ }^{+}$Huan Gao and Qin Guo contributed equally to this work.

\section{References}

[1] Li X, Jiang YH, Jiang P, Yang JL, Ma DF, Yang CH. Effect of Guizhi Decoction on heart rate variability and regulation of cardiac autonomic nervous imbalance in diabetes mellitus rats. CHIN J INTEGR MED 2014;20:524-33.

[2] Du X L, Sui F, Huo HR, Zhang HW, Luo K, Li LF, et al. Reciprocal effects of Guizhi decoction to the Guizhi decoction syndrome by toll-like receptor mRNA expression and cytokines secretion. CHIN J 
INTEGR MED 2013;19:826-35.

[3] Wu XX, Zhang LS, Gao H, Song JN, Bai D. Antipyretic Mechanism of Guizhitang and Its Effective Components Based on Network Pharmacology. Chin J Exp Tradit Med Formulae 2018;24:190-7.

[4] Chen YR, Gao CL, Ma YM, Qiu FR. Pharmacokinetic study of multiple active constituents after oral gavage of Guizhi decoction in rats using a LC-MS/MS method. Eur J Drug Metab Pharmacokinet 2013;38:283-93.

[5] Huo H, Tan Y, Zhou A. Effect and mechanism of active fraction a guizhi decoction on dualdirectional thermoregulation: effect on heat shock protein in hypothalamus of rats. Zhongguo Zhong Yao Za Zhi 2000;10:619-21.

[6] Zhang BG, Liang XX, Liu QF. Pharmacodynamics research of Guizhi decoction in modern times. China J Chin Mater Med 2007;32:557-61.

[7] Fu HY, Zhou AX, Guo SY. Mechanism of decoction of guizhi tang on dual-directional thermoregulation. Effect on the action of bombesin. Zhongguo Zhong Xi Yi Jie He Za Zhi 1994;14:99100,70 .

[8] Sun C, Xu W, Zhang Y, Yu L, Ye M. Simultaneous Determination of 11 Compounds in Gualou Guizhi Granule and Pharmacokinetics Study by UPLC-MS/MS. J ANAL METHODS CHEM 2017;2017:1-13.

[9] Ji B, Zhuo L, Yang B, Wang Y, Li L, Yu M, et al. Development and validation of a sensitive and fast UPLC-MS/MS method for simultaneous determination of seven bioactive compounds in rat plasma after oral administration of Guizhi-gancao decoction. J Pharm Biomed Anal 2017;137:23-32.

[10] Zhao L, Xiong Z, Sui Y, Zhu H, Zhou Z, Wang Z, et al. Simultaneous determination of six bioactive constituents of Guizhi Fuling Capsule in rat plasma by UHPLC-MS/MS: Application to a pharmacokinetic study. J CHROMATOGR B 2015;1001:49-57.

[11] Chang XY, Guo GM, Fan Z, Wang HL, Liu Y, Han LJ. Simultaneous determination of two bioactive components of Huangqi Guizhi Wuwu Decoction in rat plasma using UPLC-MS/MS and its application to a pharmacokinetic study. J Chin Pharm Sci 2018;27:263-72.

[12] Xiang HJ, Zhang LS, Song JN, Fan B, Bai D. The Profiling and Identification of the Absorbed Constituents and Metabolites of Guizhi Decoction. INT J MOL SCI 2016;17:1049.

[13] Askari F, Rashidkhani B, Hekmatdoost A. Cinnamon may have therapeutic benefits on lipid profile, liver enzymes, insulin resistance, and high-sensitivity C-reactive protein in nonalcoholic fatty liver disease patients. NUTR RES 2014;34:143-8.

[14] Pires RH, Montanari LB, Martins CHG, Zaia JE, Almeida AMF, Matsumoto MT, et al. Anticandidal Efficacy of Cinnamon Oil Against Planktonic and Biofilm Cultures of Candida parapsilosis and Candida orthopsilosis. MYCOPATHOLOGIA 2011;172:453-64.

[15] Wang QS, Gao T, Cui YL, Gao LN, Jiang HL. Comparative studies of paeoniflorin and albiflorin from Paeonia lactiflora on anti-inflammatory activities. PHARM BIOL 2014;52:1189-95.

[16] Jiang C, Xu L, Chen L, Han Y, Tang J, Yang Y, et al. Selective suppression of microglial activation by paeoniflorin attenuates morphine tolerance. EUR J PAIN 2015;19:908-19.

[17] Min ZWSY. Paeoniflorin ameliorates ischemic neuronal damage in vitro via adenosine A1 receptormediated transactivation of epidermal growth factor receptor. ACTA PHARMACOL SIN 2015;36:298310.

[18] Nam K, Yae CG, Hong J, Cho D, Lee JH, Lee EH. Paeoniflorin, a monoterpene glycoside, attenuates lipopolysaccharide-induced neuronal injury and brain microglial inflammatory response. BIOTECHNOL LETT 2013;35:1183-9. 
[19] Xu WZ, Zhou LN, Ma XN, Chen Y, Qin B, Zhai X, et al. Therapeutic effects of combination of paeoniflorin and albiflorin from Paeonia radix on radiation and chemotherapy-induced myelosuppression in mice and rabbits. Asian Pacific journal of cancer prevention : APJCP 2011;12:2031.

[20] Lu P, Chen Y, Tan M, Wu Y. Chemical profiling by LC-MS/MS and HPLC fingerprint combined with chemometrics and simultaneous determination of 16 characteristic ingredients for the quality consistency evaluation of Shaoyao-Gancao Decoction. BIOMED CHROMATOGR 2018:e4401.

[21] Kaur P, Kaur S, Kumar N, Singh B, Kumar S. Evaluation of antigenotoxic activity of isoliquiritin apioside from Glycyrrhiza glabra L. TOXICOL IN VITRO 2009;23:680-6.

[22] Asl MN, Hosseinzadeh H. Review of pharmacological effects of Glycyrrhiza sp. and its bioactive compounds. PHYTOTHER RES 2008;22:709-24.

[23] Akutagawa K, Fujita T, Ouhara K, Takemura T, Tari M, Kajiya M, et al. Glycyrrhizic acid suppresses inflammation and reduces the increased glucose levels induced by the combination of Porphyromonas gulae and ligature placement in diabetic model mice. INT IMMUNOPHARMACOL 2018;68:30-8.

[24] Fan B, Jiang B, Yan S, Xu B, Huang H, Chen G. Anti-Inflammatory 18 \& -Glycyrrhetinin Acid Derivatives Produced by Biocatalysis. PLANTA MED 2018;85:56-61.

[25] Kamisli S, Ciftci O, Taslidere A, Basak TN, Ozcan C. The beneficial effects of 18betaglycyrrhetinic acid on the experimental autoimmune encephalomyelitis (EAE) in C57BL/6 mouse model. Immunopharmacol Immunotoxicol 2018;40:344-52.

[26] Liu X, Ma X, Huo C, Yu S, Wang Q. [Microbiological transformation of paeoniflorin and albiflorin]. Zhongguo Zhong Yao Za Zhi 2010;35:872-5.

[27] Wang K, Liu L, Yang Y, Liu X, Zhang L, Xu W, et al. An effective UFLC-MS/MS method used to study pharmacokinetics of major constituents of Fukeqianjin formula in rat plasma. Chin Med 2020;15:74. 
Figures

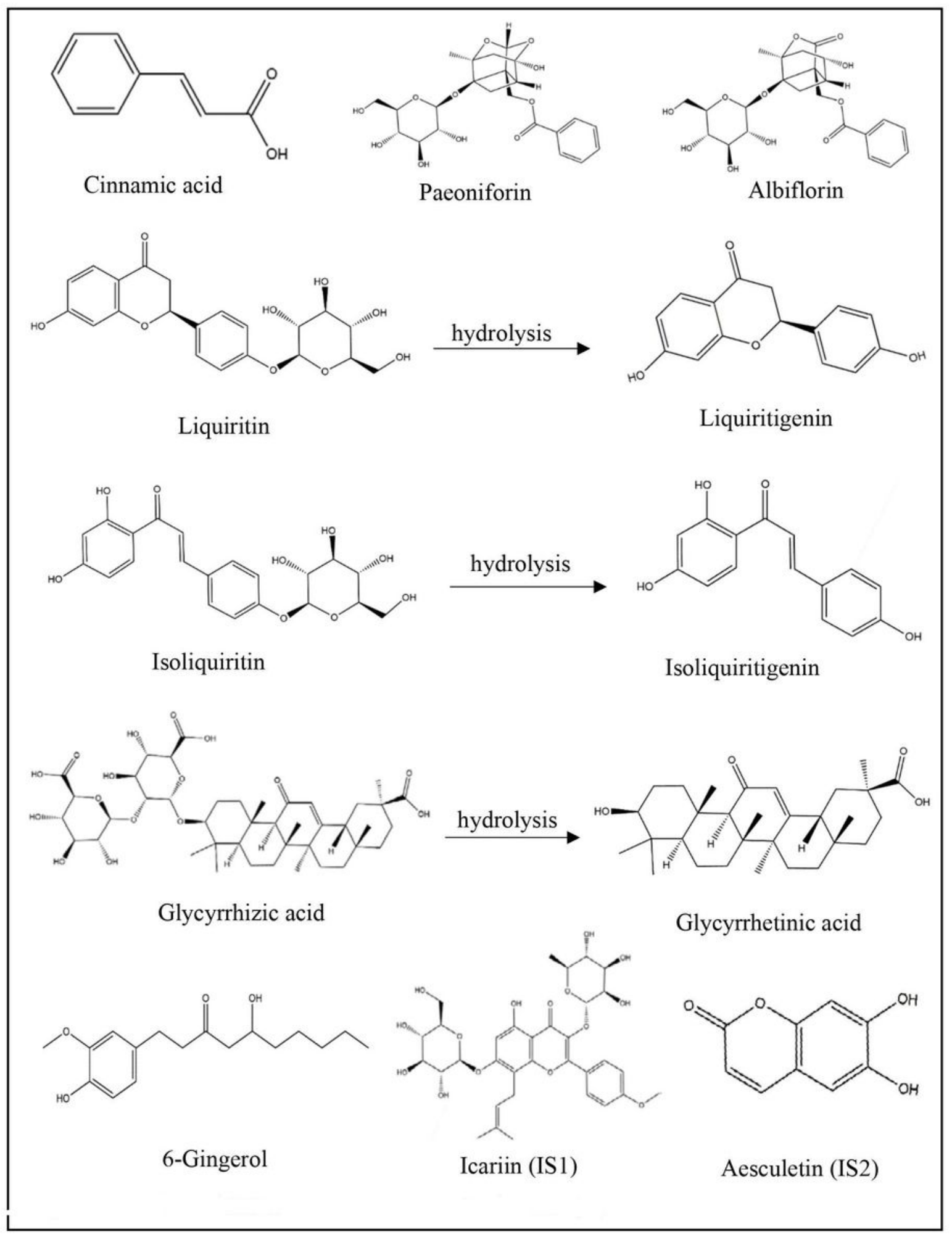

Figure 1

Chemical structures of analytes and internal standard (IS) 
$\times 10$

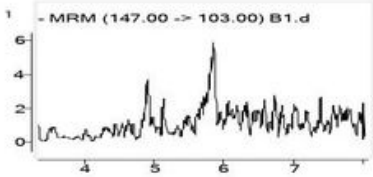

- MRM (525.20 $\rightarrow$ 121.00) Blank.d

$\times 10$

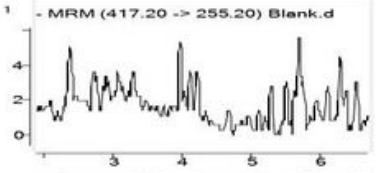

$\times 10$ 1. - MRM (417.20 -> 255.20) Blank.d

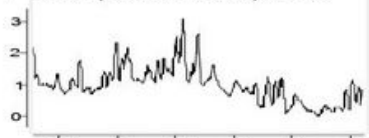

$\times 10=$ - MRM $(255.10 \rightarrow 119.00)$ Biankd

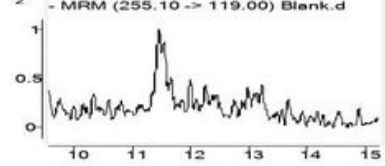

$\times 102$ - MRM ( 821.20 -> 350.90) Blank.d

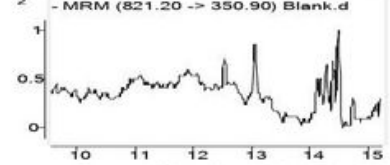

$\times 10=-$ MRM (469.40 $>$ 425.50) Blank.d

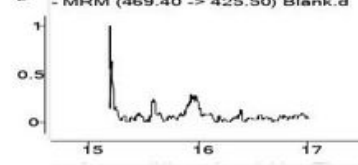

$\times 102-\operatorname{MRM}(293.10 \rightarrow 99.10)$ B1.d

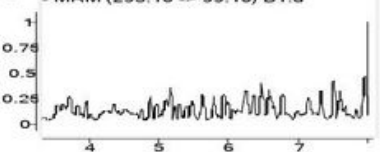

$\times 10=-\operatorname{MRM}(721.30 \rightarrow 367.20)$ Blank.d

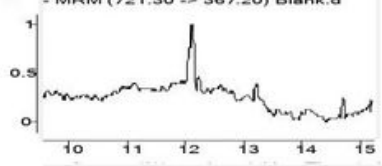

$\times 10=-\operatorname{MRM}(177.10 \rightarrow 133.00) 180513010$

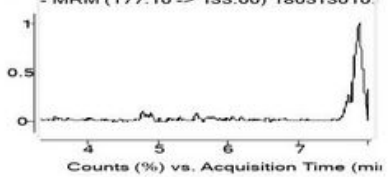

A
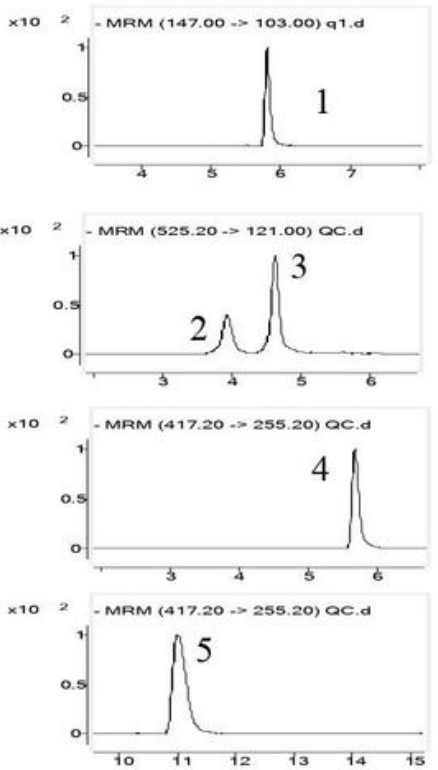

$\times 102-$ MRM (255.10 $>$ 119.00) QC.d
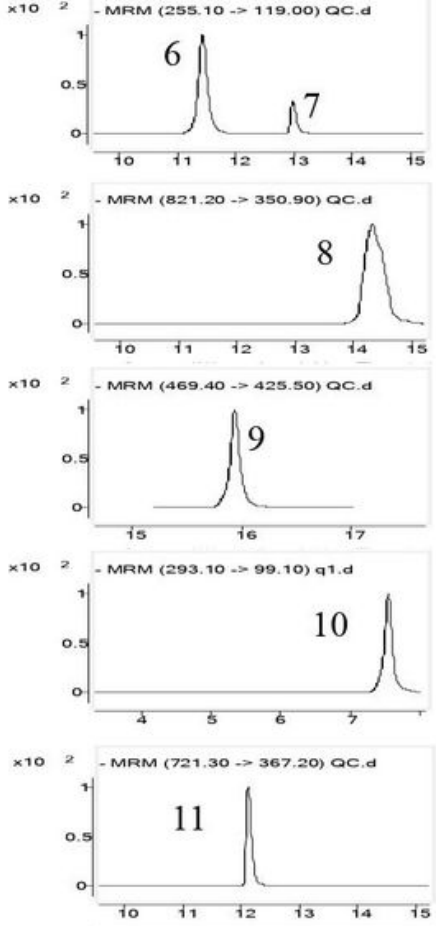

$\times 10$

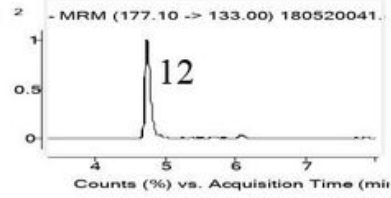

B
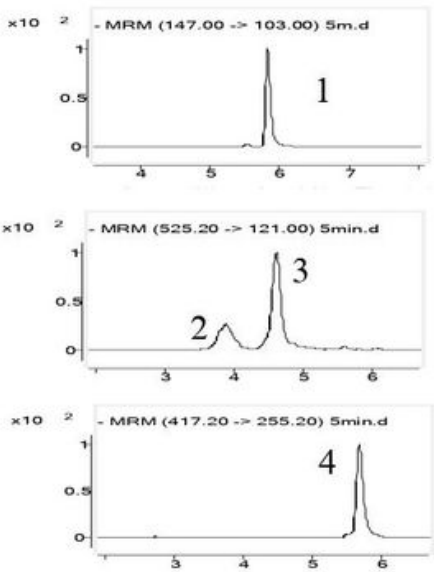

$\times 102-M R M(417.20->255.20) 5 \mathrm{~min}$
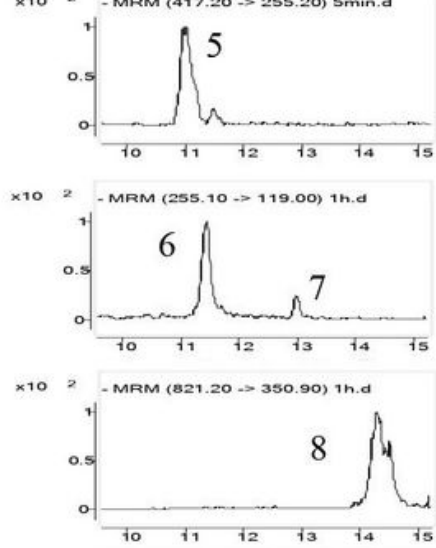

$\times 10=-M R M(469.40 \rightarrow 425.50)$ sh.d
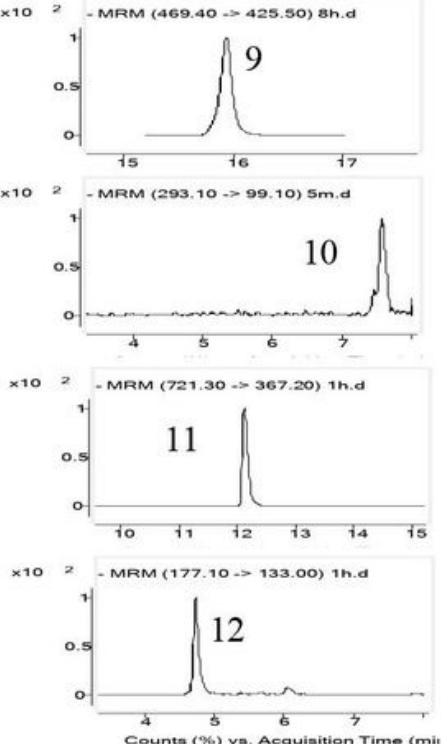

C $\times 10^{2}-M P M(147.00-103.00) 180521004$
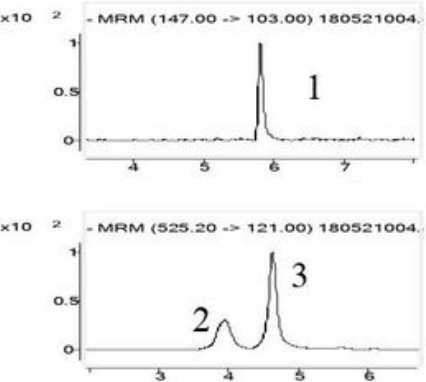

$\times 10=-M R M(417.20=->255.20) 180521004$

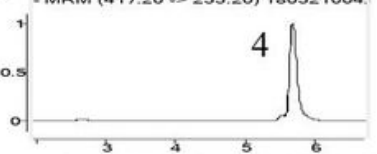

$\times 10=$ MRM $(417.20 \rightarrow 255.20) 180521004$

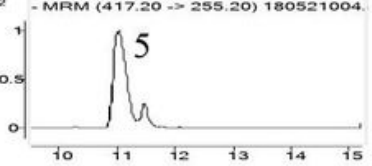

$\times 10=$-MRM(255.10 -> 119.00) 180521004
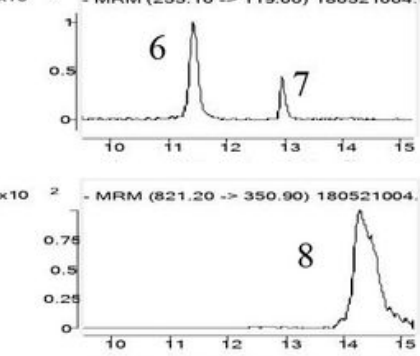

$\times 10=2$ MPM (469.40 $>425.50)$ 180611900:

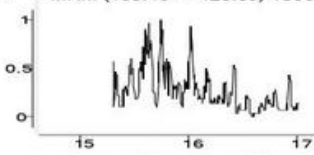

$\times 102-$ MRM $(293.10 \rightarrow 99.10) 180521004$.

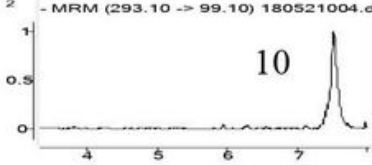

$\times 10=-\operatorname{MRM}(721.30 \rightarrow 367.20) 180521004$
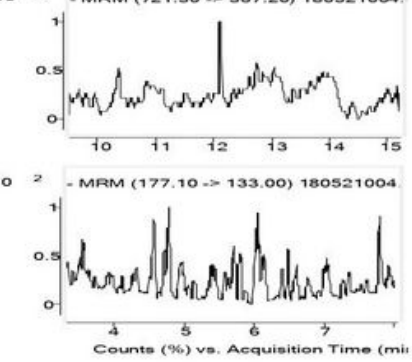

D

Figure 2

Multiple reaction monitoring mode (MRM) chromatograms of cinnamic acid (1), paeoniflorin (2), albiflorin (3), liquiritin (4), isoliquiritin (5), liquiritigenin (6), isoliquiritigenin (7), glycyrrhizic acid (8), glycyrrhetinic acid (9), 6-gingerol (10), icariin (11; IS1), and aesculetin (12; IS2) . blank rat serum (A), blank rat serum spiked with the analytes and ISs (B), rat serum samples at $5 \mathrm{~min}, 1 \mathrm{~h}$, or $8 \mathrm{~h}$ after oral administration of GZD (C), and GZD after 1000 times dilution with methanol(D). 

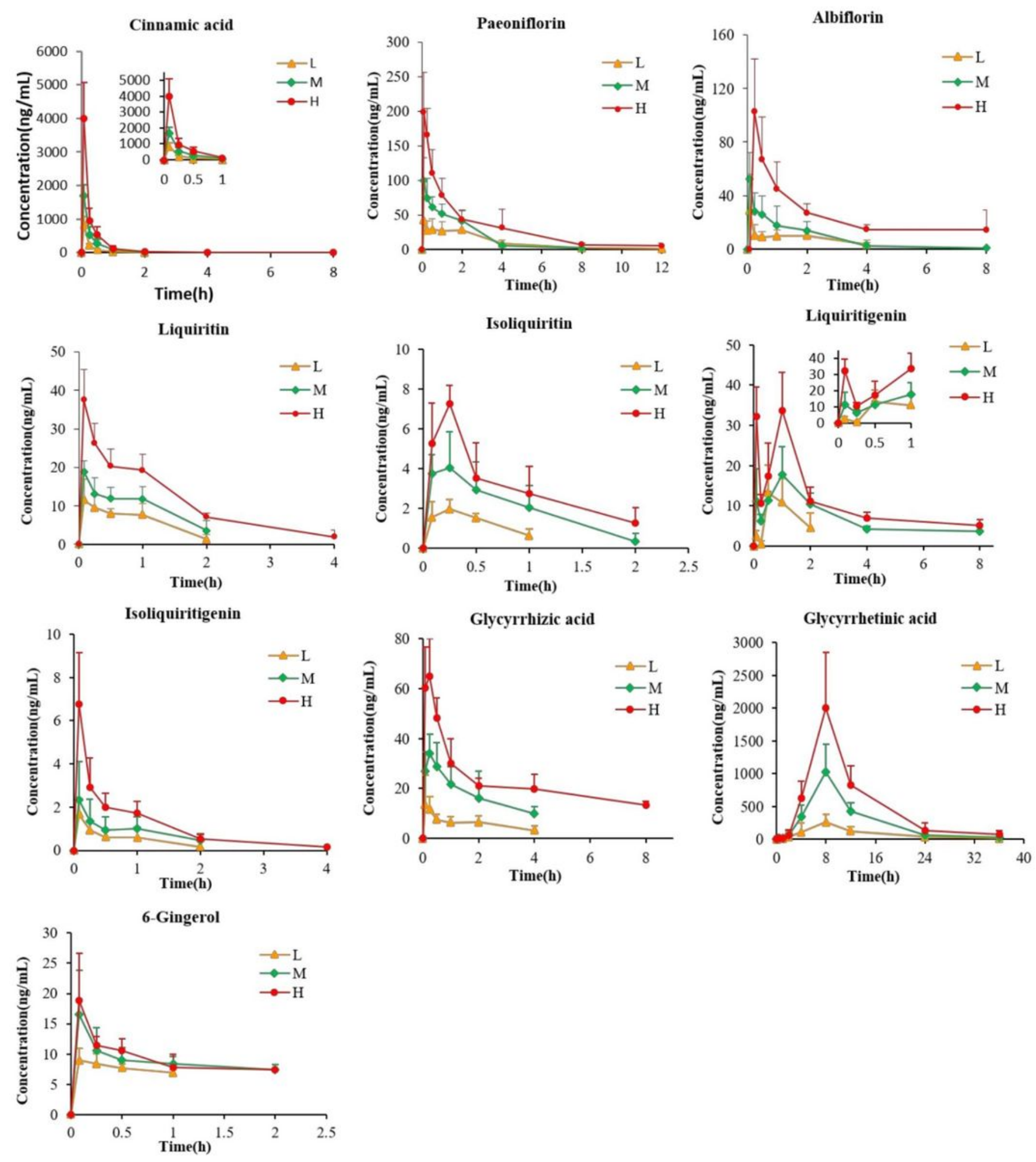

\section{Figure 3}

Mean blood concentration-time curves of the 10 analytes in rat plasma after oral administration of GZD ( $\mathrm{H}$ : high dose, $40.0 \mathrm{~g}$ crude drug $\cdot \mathrm{kg}-1$, M: medium dose, $20.0 \mathrm{~g}$ crude drug $\cdot \mathrm{kg}-1$, L: low dose, $10.0 \mathrm{~g}$ crude drug $\cdot \mathrm{kg}-1$; each point represents mean $+S D, n=6$ ). 

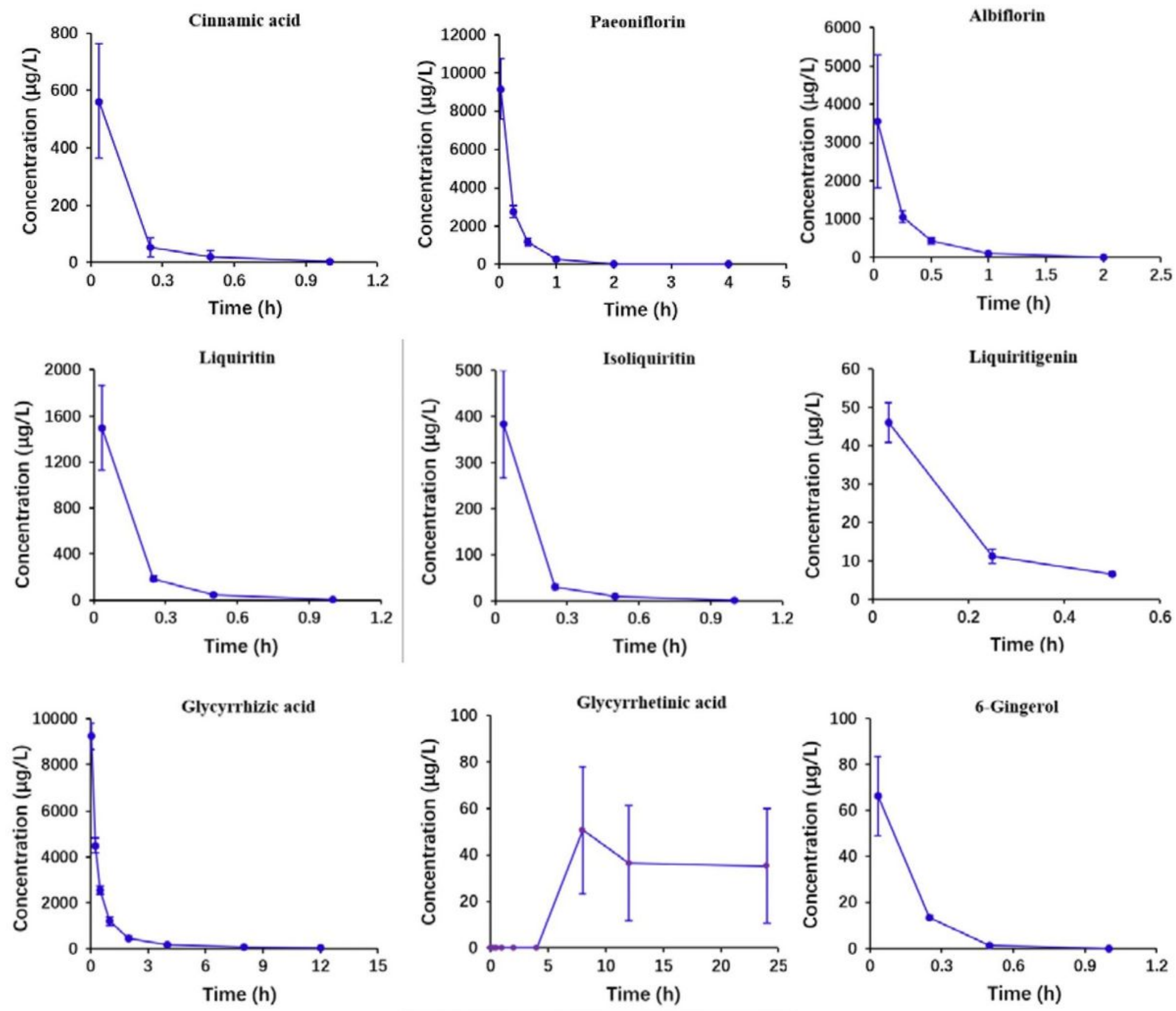

\section{Figure 4}

Mean blood concentration-time curves of the 9 analytes in rat plasma after intravenous injection of GZD at a dose of $2.0 \mathrm{~g}$ crude drug $\cdot \mathrm{kg}-1$ (each point represents mean $\pm S D, n=5$ ). 